\title{
The Inability of the Choroid to Revascularize in Oxygen-Induced Retinopathy Results from Increased p53/miR-Let-7b Activity
}

Tianwei E. Zhou, ${ }^{\star \dagger}$ Tang Zhu ${ }^{\ddagger}$ José C. Rivera, ${ }^{\dagger \ddagger}$ Samy Omri, ${ }^{\dagger}$ Houda Tahiri, ${ }^{\dagger}$ Isabelle Lahaie, ${ }^{\dagger}$ Raphaël Rouget, ${ }^{\ddagger}$ Maëlle Wirth, Stanley Nattel, ${ }^{*}$ Gregory Lodygensky, Gerardo Ferbeyre, ${ }^{* *}$ Mohammad Nezhady, ${ }^{\dagger \dagger}$ Michel Desjarlais, ${ }^{* \dagger}$ Patrick Hamel, ${ }^{\S}$ and Sylvain Chemtob ${ }^{*}+\S \S$

From the Department of Pharmacology and Therapeutics, ${ }^{*}$ McGill University, Montréal, Québec; and the Department of Ophthalmology, ${ }^{\dagger}$ MaisonneuveRosemont Hospital Research Centre, the Departments of Pharmacology, ${ }^{\ddagger}$ Ophthalmology, ${ }^{\S}$ and Pediatrics, ${ }^{\|}$Sainte-Justine University Hospital Centre, the Department of Medicine, Montreal Heart Institute, and the Departments of Biochemistry** and Pathology and Cell Biology, ${ }^{\dagger \dagger}$ University of Montréal, Montréal, Québec, Canada

Accepted for publication July 26, 2019.

Address correspondence to Sylvain Chemtob, M.D., Ph.D., or Tianwei E. Zhou, M.D., Ph.D., Department of Ophthalmology, MaisonneuveRosemont Hospital Research Centre, University of Montreal, 5415 Blvd de l'Assomption, Montreal, QC H1T 2M4, Canada. E-mail: sylvain.chemtob@ umontreal.ca or ellen.zhou@ umontreal.ca.
Retinopathy of prematurity (ROP) is characterized by an initial retinal avascularization, followed by pathologic neovascularization. Recently, choroidal thinning has also been detected in children formerly diagnosed with ROP; a similar sustained choroidal thinning is observed in ROP models. But the mechanism underlying the lack of choroidal revascularization remains unclear and was investigated in an oxygeninduced retinopathy (OIR) model. In OIR, evidence of senescence was detected, preceded by oxidative stress in the choroid and the retinal pigment epithelium. This was associated with a global reduction of proangiogenic factors, including insulin-like growth factor 1 receptor (Igf1R). Coincidentally, tumor suppressor p53 was highly expressed in the 0IR retinae. Curtailing p53 activity resulted in reversal of senescence, normalization of Igf1r expression, and preservation of choroidal integrity. OIR-induced downregulation of Igf1r was mediated at least partly by miR-let-7b as i) let-7b expression was augmented throughout and beyond the period of oxygen exposure, ii) let-7b directly targeted Igf1r mRNA, and iii) p53 knock-down blunted let-7b expression, restored Igf1r expression, and elicited choroidal revascularization. Finally, restoration of Igf1r expression rescued choroid thickness. Altogether, this study uncovers a significant mechanism for defective choroidal revascularization in OIR, revealing a new role for p53/let7b/IGF-1R axis in the retina. Future investigations on this (and connected) pathway could further our understanding of other degenerative choroidopathies, such as geographic atrophy. (Am J Pathol 2019, 189: 2340-2356; https://doi.org/10.1016/j.ajpath.2019.07.009)
Retinopathy of prematurity (ROP) is the leading cause of severe visual impairment and blindness in neonates and young children in North America and Europe. ${ }^{1}$ ROP has long been associated with damage of the inner retina, including dysregulated angiogenesis of retinal vessels. ${ }^{2}$ Recently, clinical and preclinical animal studies have detected choroidal thinning in subjects previously afflicted with $\mathrm{ROP}^{3-6}$ (Supplemental Figure S1), which can result in photoreceptor injury. ${ }^{7,8}$ But unlike retinal vessels that undergo neovascularization on retinal ischemia and hypoxia, the choroid - the exclusive source of oxygen and nutrients to photoreceptors ${ }^{9}-$ does not revascularize and remains thin despite ensuing local hypoxia. ${ }^{7}$ The underlying mechanisms for such paradoxical persistent choroidal thinning remain unexplained.

Supported by the Canadian Institutes of Health Research M.D./Ph.D. Scholarship, the Suzanne Véronneau-Troutman Fund, and the University of Montreal Ophthalmology Research Fund (T.E.Z.); the Heart and Stroke Foundation of Canada and the Canadian Stroke Network (J.C.R.); and Canadian Institutes of Health Research grant MOP12532, the March of Dimes Birth Defects Foundation, the Quebec Health Research Fund, and the Canada Research Chair (Vision Science) and the Leopoldine Wolfe Chair in Translational Research in Age-Related Macular Degeneration (S.C.).

Disclosures: None declared. 
Along these lines, early senescence is a process that promotes age-related disorders; and when applied to cell replication, it interferes with proliferation. In this context, oxidative stress and hyperoxia, as well as hypoxia and nutrient deficiency to the retinal pigment epithelium (RPE) and photoreceptors secondary to choroidal involution, ${ }^{7}$ are established triggers of senescence. ${ }^{10}$ Oxygen supplementation and deficient antioxidant defense in premature subjects exert a major contribution in the development of ROP/ oxygen-induced retinopathy (OIR). ${ }^{2}$ Ensued increased generation of reactive oxygen species from the oxidative stress readily induces DNA damage ${ }^{10}$ and subsequently activates ataxia-telangiesctasia mutated serine/threonine kinase kinase-a potent activator of p53. ${ }^{11}$ Hypoxic ischemia, at times linked to oxidative stress, elicits an endoplasmic reticulum stress and unfolded protein response to limit protein translation, which concomitantly increases degradation of mRNA through activation of inositol-requiring enzyme $1 \alpha$ endoribonuclease, followed by induction of p53 $3^{12,13}$ contributing to the senescent phenotype. ${ }^{14}$ Through its properties as a transcription factor and others, ${ }^{15}$ p53 tightly regulates cell fate, including cell cycle progression, apoptosis, and senescence-all aiming to maintain genomic stability and avoid passage of DNA damages to daughter cells. $^{16}$ In particular, p53 governs replicative cellular senescence by targeting p21/cyclin-dependent kinase inhibitor 1 and different miRNAs. ${ }^{17}$

The expression of p53 is largely regulated by miRNAs ${ }^{18}$ and, in turn, p53 controls the expression of various miRNAs involved in cell fate and angiogenesis. ${ }^{19}$ miRNAs are a family of noncoding RNAs that fine-tune post-transcriptional regulation of genes. On the basis of knowledge mostly acquired from cancer research, p53 regulates angiogenic pathways via certain families of miRNAs - for instance, the p53/miR-200 pair targets the vascular endothelial growth factor (VEGF) pathway, ${ }^{20,21}$ whereas p53/ let-7 modulates the critical insulin-like growth factor 1 (IGF1) pathway at multiple levels. ${ }^{22-24}$ But in retinal diseases, mechanistic insights about the interactions of p53, miRNAs, and their downstream pathways are scarce. Herein, we propose to investigate the unexplored role of p53 and regulatory miRNAs in contributing to deficient choroidal revascularization in the context of ROP. This study also explores the plausible link between cellular stress experienced in the neonatal period with early senescence.

\section{Materials and Methods}

\section{Animals}

Pigmented Long-Evans rats were purchased from Charles Rivers Laboratories (Saint-Constant, Canada). All experiments were conducted in accordance with the Association for Research in Vision and Ophthalmology statement regarding use of animals in ophthalmic and vision research and were approved by the Hôpital Maisonneuve-Rosemont Animal Care Committee.

\section{Using Optical Coherence Tomography to Measure Choroidal Thickness}

A portable, noncontact, hand-held, high-resolution optical coherence tomography instrument (Envisu C2300; Leica Microsystems, Wetzlar, Germany) was used. Patients were imaged in the neonatal intensive care unit at Sainte-Justine Hospital during ROP screening. Informed consent for publication was obtained from parents or legal guardians.

Patients were imaged without the use of anesthetic or eyelid speculum. Choroidal thickness, from the posterior edge of the RPE to the choroid/sclera junction, was measured with the built-in caliper, applied perpendicularly to the RPE, just under the fovea.

\section{OIR Model}

Newborn Long-Evans rats were placed with their mothers under oxygen concentration, which cycles at $50 \% \pm 1 \%$ or $10 \% \pm 1 \%$ every 24 hours from postnatal day (P) 0 to P14. On P14, all rats were returned to room air. This model is characterized by a retinal avascularization predominantly in the periphery (at P14), followed by hypoxia-driven retinal neovascularization clearly present at P18, which mimics the two principal phases of ROP in humans. ${ }^{25,26}$ Controls were maintained in room air $\left(21 \% \mathrm{O}_{2}\right)$. In some cases, pups were selected to receive twice-daily i.p. injections of p53 inhibitor [pifithrin- $\alpha$ (PFT $\alpha$ ); $20 \mathrm{mg} / \mathrm{kg}$; Sigma-Aldrich, St. Louis, MO] from P0 to P14. Animals were sacrificed at different ages (P3, P6, P10, P14, P18, and P30), and eyes were collected and processed for measurements described below.

\section{Separation of RPE Cells and Choroid}

Pigmented Long-Evans rats facilitated dissection of RPE cells from choroid. Rats were sacrificed at various ages (P3 to P30, as described above), and eyes were enucleated. Eyes were washed in icy phosphate-buffered saline (catalog number 311-012-CL; Wisent Inc., Saint-Jean-Baptiste, Canada) with gentle shaking for 10 minutes to remove any attached animal hair. The cornea and lens were subsequently removed. The freshly isolated eye cups, which contained neuroretina, RPE, choroid, and sclera, were incubated in Hanks' balanced salt solution (catalog number 14025076; Thermo Fisher Scientific, Waltham, MA) with 2.5\% TrypLE Express Enzyme (catalog number 126040132; Thermo Fisher Scientific) and $2 \mathrm{mg} / \mathrm{mL}$ Collagenase D (catalog number 11088866001; Roche Life Science, Branford, CT) at $37^{\circ} \mathrm{C}$ for 45 minutes. Eye cups were washed using Dulbecco's modified Eagle's medium (DMEM)/Nutrient Mixture F-12 (catalog number 319-075CL; Wisent Inc.) to finalize the digestion step and then kept 
on ice. The neuroretina immersed in DMEM/Nutrient Mixture F-12 was separated from RPE/choroid complex using a pair of blunt-headed forceps to gently tap on the choroid and dislodge RPE cells; then, RPE cells were recovered using a pipette. RPE cells from four eyes were pooled and centrifuged. DMEM/Nutrient Mixture F-12 solution was removed and RiboZol (catalog number N580200ML; Amresco, Solon, OH) was added to RPE cells for RNA isolation (see below).

\section{RNA Isolation and Real-Time Quantitative PCR}

Eyes were rapidly enucleated and placed into a sterile Petri dish resting on ice. The RPE/choroid complex was promptly separated from neuroretina. Both tissue complexes were processed for RNA using RiboZol, followed by treatment with DNase I (catalog number AMPD1; Sigma-Aldrich) to remove any contaminating genomic DNA. The DNasetreated RNA was then converted into cDNA using iScript Reverse Transcription Kit (catalog number 170-8841; BioRad, Mississauga, Canada). PCR primers targeting rat were designed using National Center for Biotechnology Information Primer Blast; detailed sequences are summarized in Table 1. Quantitative analysis of gene expression was generated using an ABI 7500 Real-Time PCR (Applied Biosystems, Foster City, CA) sequence detection system and the SYBR Green Master Mix Kit (catalog number 1725271; BioRad). Gene expression was calculated relative to $18 \mathrm{~S}$ universal primer pair (catalog number AM1718;

Table 1 qPCR Primer List

\begin{tabular}{ll}
\hline Gene symbols & Sequences \\
\hline Cdkn1a & F: 5'-AGTATGCCGTCGTCTGTTCG-3' \\
Tp53 & R: 5'-GAGTGCAAGACAGCGACAAG-3' \\
Nfe2l2 & F: 5'-ACAGCGTGGTGGTACCGTAT-3' \\
& R: 5'-GGAGCTGTTGCACATGTACT-3' \\
Sod1 & F: 5'-TTGTAGATGACCATGAGTCGC-3' \\
& R: 5'-CTCCATGTCCTGCTGTATGC-3' \\
Sod2 & F: 5'-CGGATGAAGAGAGGCATGTT-3' \\
& R: 5'-CAATCACACCACAAGCCAAG-3' \\
Nox1 & F: 5'-GCGACCTACGTGAACAATCT-3' \\
& R: 5'-CAGCAACTCTCCTTTGGGTT-3' \\
Nox2 & F: 5'-CCCTTTGCTTCCTTCTTGAAATC-3' \\
& R: 5'-GCACCCGTCTCTCTACAAATCC-3' \\
Nox3 & F: 5'-TGATCATCACATCCTCCACCAA-3' \\
& R: 5'-GATGGCAAGGCCGATGAA-3' \\
Nox4 & F: 5'-GCAGCATTGGCGTGTTCTT-3' \\
& R: 5'-GAAATGAACGCCCCTAGGATCT-3' \\
Igf1r & F: 5'-CTGCATCTGTCCTGAACCTCAA-3' \\
& R: 5'-TCTCCTGCTAGGGACCTTCTGT-3' \\
Igf1 & F: 5'-TGGCAGAACTGCTGTCTGAG-3' \\
& R: 5'-AACGCAGGGTCTAGTTGAGC-3' \\
& F: 5'-GCATTGTGGATGAGTGTTGC-3' \\
& R: 5'-GGTCTTGTTTCCTGCACTTC-3'
\end{tabular}

$F$, forward; $\mathrm{PPCR}$, real-time quantitative $P C R ; R$, reverse.
Ambion, Waltham, MA) expression using the $\Delta \mathrm{C}_{\mathrm{T}}$ method. Data were quantified by $\Delta \Delta \mathrm{C}_{\mathrm{T}}$ method.

\section{PCR Array}

RNA was isolated from RPE/choroid complex using the RiboZol method described in Separation of RPE Cells and Choroid and RNA Isolation and Real-Time Quantitative $P C R$. RNA was then converted into cDNA using iScript Reverse Transcription Kit (catalog number 170-8841; Bio$\mathrm{Rad}$ ). Real-time PCR for angiogenic gene profiling was performed using the SYBR Green-Based Rat Angiogenesis PCR Array (catalog number PARN-024Z; Qiagen, Hilden, Germany).

\section{miRNA PCR Array}

RNA contents were isolated using miRNeasy Mini Kit (catalog number 217004; Qiagen), as per manufacturer's instruction. Total RNA (250 ng) was reverse transcribed with the miScript II RT kit (catalog number 218161; Qiagen), according to manufacturer's guidelines. cDNA (20 $\mu \mathrm{L})$ was diluted with RNase-free water before PCR. Realtime PCR for mature miRNA expression profiling was developed using the SYBR Green-based rat hypoxia signaling pathway miScript miRNA PCR array (catalog number MIRN-121ZA; Qiagen); miRNA primers are shown in Table 2. The data obtained were analyzed using the $\Delta \Delta \mathrm{C}_{\mathrm{T}}$ method of relative quantification for miScript miRNA PCR arrays with Qiagen online software (https:// www.qiagen.com/at/shop/genes-and-pathways/data-analysiscenter-overview-page). Fold changes in miRNA between normoxia- and OIR-raised rats, with $P<0.05$, were considered statistically significant; miRNAs with statistically significant fold changes were represented separately.

\section{Microvascular Sprouting from Choroid Explants}

Choroid from juvenile rats was prepared, as previously described. ${ }^{27}$ Briefly, choroid was cut into approximately $2-\times 1-\mathrm{mm}$ pieces and placed in Matrigel (catalog number 354230; Corning, Corning, NY) with $2 \%$ fetal bovine serum and incubated in $37^{\circ} \mathrm{C}, 5 \% \mathrm{CO}_{2}$ for 48 hours before treatment. Choroidal tissue was obtained from control (normoxia) and OIR animals. For each type of in vivo condition, the left eye was cultured in normoxia for 48 hours

Table 2 miRNA qPCR Primer List

\begin{tabular}{ll}
\hline Official symbol & Gene symbols \\
\hline miRlet7b & MS00000007 \\
miRlet7d & MS00012915 \\
miR200a & MS000000581 \\
miR203 & MS00000602 \\
miR29b-1 & MS00005544 \\
\hline
\end{tabular}

qPCR, real-time quantitative $P C R$. 
in EGM-2MV medium (catalog number CC-4147; Lonza, Walkersville, MD), and the right eye was first placed in hypoxia $\left(5 \% \mathrm{O}_{2}\right)$ for 24 hours and then returned to normoxia for an additional 48 hours (also in EGM-2MV medium); in each case, tissues were cultured in presence or absence of 25 $\mu \mathrm{mol} / \mathrm{L}$ p53 inhibitor PFT $\alpha$.

Images of individual explants were taken before and after treatments. The vascular sprouting covered areas were quantified with ImageJ bundled with Java version 1.8.0_172 https://imagej.nih.gov/ij/download.html; developed by Wayne Rasband, NIH, Bethesda, MD).

\section{Ocular Tissue Preparation for Immunohistochemistry}

Animals were perfused with phosphate-buffered saline and 4\% paraformaldehyde (catalog number P6148; SigmaAldrich). For histology sections, eyes were immediately collected, dehydrated with alcohol, and embedded in paraffin. Sagittal sections (5 $\mu \mathrm{m}$ thick) were cut using a microtone (RM 2145; Leica Biosystems, Wetzlar, Germany). Eyes for cryopreparation were further fixed in $4 \%$ paraformaldehyde overnight. Posterior eye cups were frozen in OCT medium (catalog number 3801480; Leica Biosystems). Samples were then cut into sagittal sections (10 $\mu \mathrm{m}$ thick; CM3050S; Leica Biosystems) and processed for immunohistochemistry.

\section{Choroidal Thickness Measurement}

To measure choroidal thickness, cryosection samples of retina/choroid were stained with tetramethylrhodamineconjugated lectin endothelial cell marker (dilution 1:200; Sigma-Aldrich; L5264). Choroidal images were then captured by confocal microscope (Olympus, Richmond Hill, Canada). Starting from the optic nerve (the $0 \mu \mathrm{m}$ position), choroidal thickness was measured at $150-\mu \mathrm{m}$ intervals across the choroid; measurements were averaged and plotted into a spider graph. Because the rat eye changes size from P14 to P210, the thickness was measured at one-quarter of the entire choroidal length, starting from the optic nerve, which corresponds to the region most affected by OIR. ${ }^{3-6}$

\section{Western Blot Analysis}

Protein extracts $(40 \mu \mathrm{g})$ were loaded, separated by voltage potential, and transferred to polyscreen polyvinylidene difluoride transfer membrane (catalog number 1620177; BioRad), as previously reported. ${ }^{28}$ Respective proteins were revealed by antibodies listed in Table 3 .

\section{DNA Fragmentation Labeling}

Labeling of fragmented DNA was performed by using a commercial kit based on terminal deoxynucleotidyl transferase-mediated dUTP nick end labeling technique
Table 3 Antibody List

\begin{tabular}{|c|c|c|}
\hline Protein & Company (catalog number) & Dilution used \\
\hline$\beta$-Actin & $\begin{array}{l}\text { Novus Biologicals, Oakville, } \\
\text { Canada } \\
\text { (NB600-501) }\end{array}$ & $1: 4000$ \\
\hline IGF-IR $\beta$ & $\begin{array}{l}\text { Santa Cruz Biotechnology, } \\
\text { Dallas, TX (SC-713) }\end{array}$ & $1: 100$ \\
\hline $\begin{array}{c}\text { Phos-IGF-IR } \beta \\
\text { (Tyr 1161) }\end{array}$ & $\begin{array}{l}\text { Santa Cruz Biotechnology } \\
\quad(\text { SC-101703) }\end{array}$ & $1: 100$ \\
\hline p53 & $\begin{array}{l}\text { Santa Cruz Biotechnology } \\
\quad(\text { SC-393031) }\end{array}$ & $1: 100$ \\
\hline
\end{tabular}

IGF-IR $\beta$, insulin-like growth factor 1 receptor $\beta$; Phos-IGF-IR $\beta$, phosphorylated IGF-IR $\beta$.

(catalog number 11684809910; Roche Life Science, Branford, CT).

\section{siRNA Preparation}

siRNAs were synthesized using an siRNA Construction Kit (catalog number AM1620; Ambion). The target sequences of the human p53-siRNA were designed by an siRNA design tool (Chang Bioscience, Fremont, CA) based on the human p53 DNA sequence (https://www.ncbi.nlm.nih.gov/ nuсcore; accession number NM_000546.5).

Three oligonucleotides of human $\mathrm{p} 53$ were selected as the target area, which were as follows: p53-siRNA70 [sense, 5'-GAAACTTCTTCCTCCAGATGA-3' (forward)], p53siRNA741 [sense, 5'-GGCCCATCCTTACCATCATCA-3' (forward)], and p53-siRNA969 [sense, 5'-GAGAATATTTCACCCTTAAGA-3' (forward)]. For the siRNA hairpin loop construction, AA and CCTGTCTC codes were added into the $5^{\prime}$ - and $3^{\prime}$-terminal of the selected oligonucleotides, respectively. The designed hairpin oligonucleotides were then synthesized by Alpha-DNA Inc. (Montreal, Canada).

The synthesized siRNA hairpin oligonucleotides were used for the siRNA synthesis on the instruction sheet of the siRNA Construction Kit (catalog number AM1620; Ambion), which underwent the processes of transcription template preparation, double-stranded RNA synthesis, and siRNA preparation and purification.

To characterize the siRNAs, a rat retinal ganglion cell line was used (retinal ganglion cell line was kindly provided by Dr. Neeraj Agarwal, National Eye Institute, Bethesda, MD). Retinal ganglion cells were transfected with siRNA70, siRNA741, and siRNA969, as previously reported ${ }^{29,30}$; and real-time quantitative PCR was performed to confirm their knock-down efficiencies.

\section{In Silico Prediction of Potential miRNA Targets}

miRNAs that can potentially target rat Igf1 receptor (Igf1r) transcript were predicted via TargetScan version 6.2 (TargetScan, Cambridge, MA) by searching for the presence of 
well-conserved 8-mer (2636 to 2643) and 7-mer (6674 to $6680)$ sites on the $3^{\prime}$-untranslated region $\left(3^{\prime} \mathrm{UTR}\right)$ of $I g f 1 r$ mRNA. PicTar (http://pictar.mdc-berlin.de; last accessed February 7, 2019) and DIANA-microT-CDS (http://www. microrna.gr/microT-CDS; last accessed February 7, 2019) were used to confirm TargetScan findings.

\section{Construction of Luciferase-miRNA-Target Site Fusion Plasmids}

The pmirGLO Dual-Luciferase miRNA Target Expression Vector (catalog number E1330; Promega, Madison, WI) was provided by S.N. To construct reporter vectors bearing miRNA-target sites, fragments containing the exact target sites for let-7b in the $3^{\prime} \mathrm{UTR}$ of rat $I g f 1 r$ mRNA were synthesized (Top Gene Technologies Inc., Montreal, Canada). Each construct contained one of the two predicted binding sites for let-7b. These inserts were ligated into PmeI and XbaI sites on the pmirGLO Dual-Luciferase miRNA Target Expression Vector.

\section{The Dual-Luciferase Reporter Assay}

The dual-luciferase assay was performed with DualLuciferase Reporter Assay System (catalog number E1910; Promega). ARPE-19 cells (catalog number CRL2302; ATCC, Manassas, VA) or rat brain microvascular endothelial cells (catalog number R840-05a; Cell Applications Inc., San Diego, CA) were seeded onto a 96-well plate at $2 \times 10^{4}$ cells/well. On the following day, $0.337 \mu \mathrm{g}$ of the reporter vector (eg, Igf1r-3'UTR-pmirGLO-1/2) with miRNA mimic Syn-rno-let-7b-5p (catalog number MSY0000775; Qiagen) (working concentration $=20$ nmol/L) was transfected into ARPE-19 cells using 1.684 $\mu \mathrm{L}$ of polyethylenimine transfection reagent (Polysciences, Warrington, PA) at $1 \mathrm{mg} / \mathrm{mL}$. AllStars Negative Control siRNA (catalog number SI03650318; Qiagen) was used as the negative control. The luciferase reporter assay was performed 48 hours after transfection. Standard protocol, provided by the manufacturer, was followed. Briefly, ARPE-19/rat brain microvascular endothelial cells were lysed with Passive Lysis Buffer (20 $\mu$ L/well) and gently shook at room temperature for 15 minutes. The assay plate was then inserted into an EnVision Microplate screening fluorescence reader (catalog number 2104-0010A; PerkinElmer, Waltham, MA). As soon as $75 \mu \mathrm{L}$ Luciferase Assay Reagent II (Promega) was injected, the firefly luciferase signal was measured at $640 \mathrm{~nm}$. Next, $75 \mu \mathrm{L}$ of the Stop \& Glo Reagent Buffer (Promega) was injected and the Renilla luciferase signal (used as an internal control) was measured at $525 \mathrm{~nm}$. The firefly OD/Renilla OD ratio was calculated. The activity of Syn-has/rno-let-7b-5p was calculated by subtracting the firefly OD/Renilla OD ratio of samples that received AllStars Negative Control miRNA from that of samples that received Syn-has/rno-let-7b.
Preparation of Rat IGF1R Lentivirus for Subretinal Injection

The basic protocol of the lentiviral plasmid transfection has been previously described ${ }^{31}$ and modified. To prepare lentiviral particles, $9 \mu \mathrm{g}$ of rat Igf1r (https://www.ncbi.nlm.nih. gov/nuccore; accession number NM_052807.2; catalog number LVP933; Genetarget, San Diego, CA) plasmid was mixed with $6 \mu \mathrm{g}$ of pRSV-Rev (Addgene, Cambridge, MA), $7.8 \mu \mathrm{g}$ of pCMV-vesicular stomatitis virus glycoprotein (Addgene), and $15 \mu \mathrm{g}$ of $\mathrm{pMDLg} /$ pRRE plasmid (Addgene), in $1.75 \mu \mathrm{L}$ DMEM (catalog number 11995065; Thermo Fisher Scientific). Polyethylenimine linear (140 $\mu \mathrm{L}$ of $1 \mathrm{mg} / \mathrm{mL}$; catalog number 23966; Polysciences Inc., Warrington, PA) in 1.61 $\mathrm{mL}$ of DMEM was added to the Igf1r plasmid mixture. Fifteen minutes later, the mixed transfection reagent was pipetted into a 15-cm-diameter culture dish preseeded with HEK293T cells at $50 \%$ to $60 \%$ confluence in $15 \mathrm{~mL}$ of complete DMEM. In total, four dishes were applied for lentiviral particle concentration. Twenty-four hours later, the transfecting medium was replaced with $18 \mathrm{~mL}$ complete DMEM. After a 30-hour incubation, the maximal secreted lentiviral particles in the medium were collected and sterilized by passing through a $0.22-\mu \mathrm{m}$ filter (catalog number GSWP01300; Merck Millipore Ltd, Co, Cork, Ireland). For subretinal injection, the fresh lentiviral particle medium was concentrated by ultracentrifugation at $50,000 \times g$ for 4 hours. The supernatant medium was carefully removed, and the remaining pellet (concentrated lentivirus particle) was resuspended in $30 \mu \mathrm{L}$ of sterilized phosphate-buffered saline by shaking $(8 \times g)$ at $4{ }^{\circ} \mathrm{C}$ for overnight. The final viral particles were gently mixed by pipetting, aliquoted into $10 \mu \mathrm{L} / \mathrm{each}$ into PCR microtubes, and stocked at $-80^{\circ} \mathrm{C}$ until use. The concentration of the purified lentiviral particles was detected by Lenti-X p24 Rapid Titer Kit (catalog number 632200; Takara, Kusatsu, Japan) on the kit menu instruction.

\section{Subretinal Injections of siRNA or Lentivirus}

Rat pups (P14) were anesthetized with $85 \mathrm{mg} / \mathrm{kg}$ ketamine (Vetalar; Bioniche Animal Health, Belleville, ON, Canada) and $5.0 \mathrm{mg} / \mathrm{kg}$ xylazine (Rompun; Bayer HealthCare, Mississauga, ON, Canada). Animals were placed at lateral recumbency under a dissecting microscope (SteREO Discovery V8; Zeiss, Toronto, Canada). The cornea was punctured slightly anterior to the ora serrata with a 23 -gauge needle (catalog number 305143; Becton Dickinson, Franklin Lakes, $\mathrm{NJ}$ ); the needle was advanced full thickness through the cornea into the anterior chamber. Extra care was taken to avoid injuring the lens. A slight resistance to the movement of the needle indicated penetration of the retina and entrance into the subretinal matrix; $1 \mu \mathrm{L}$ of siRNA or lentivirus solution was injected into central subretinal regions because choroid 
A

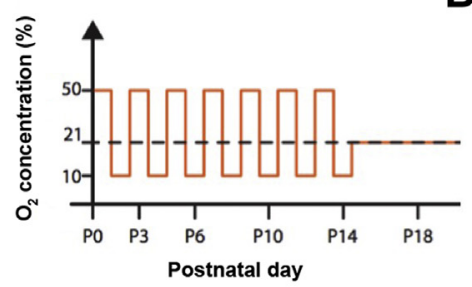

B

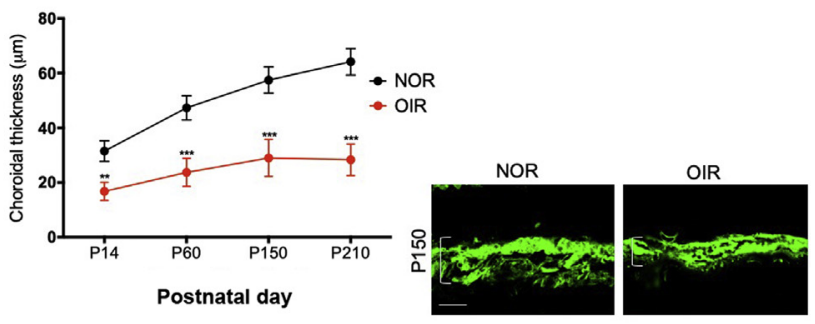

C

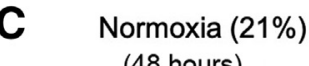

(48 hours)

NOR

Hypoxia (5\%)

(24 hours)

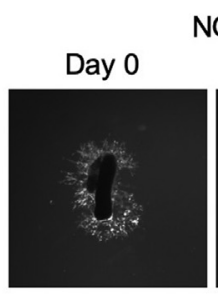

Day 2

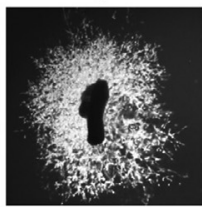

OIR

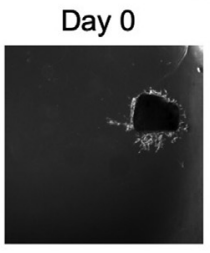

Day 2
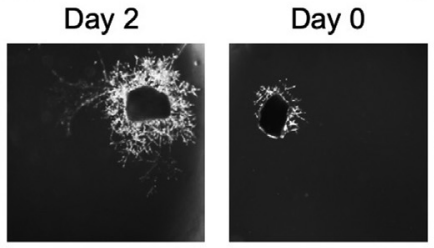

OIR

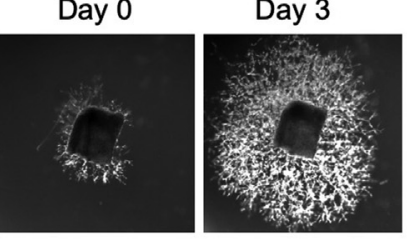

Day 3

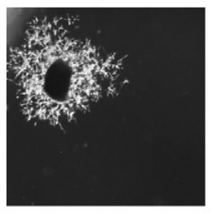

E

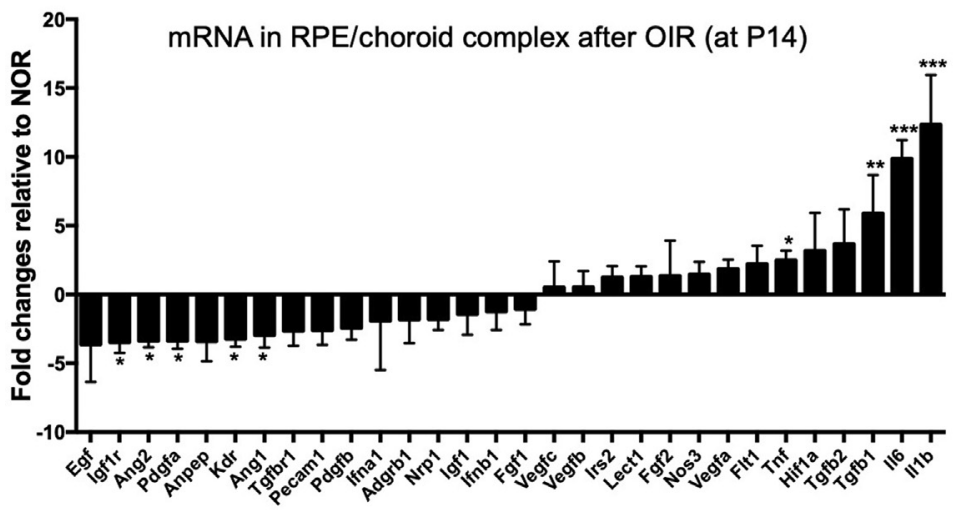

Figure 1 Choroid from oxygen-induced retinopathy (OIR)-subjected rats reveals diminished angiogenic capability. A: The cyclic OIR model was used in this study. Postnatal day $(P) 1$ rat pups were placed under alternating $\mathrm{O}_{2}$ concentrations at $50 \%$ and $10 \%$. The oxygen exposure period lasted 14 days, after which rats were returned to normoxia (NOR; indicated by the black dashed line). B: Choroidal involution in OIR animals persists beyond the oxygen exposure period into adult life. Left panel: Choroidal thicknesses of control and OIR rats were compared longitudinally. Choroids in the OIR group were significantly thinner (approximately 50\%) than the NOR controls. Right panel: A representative immunohistochemistry image of choroidal thinning in OIR. The brackets outline choroidal thickness. $P$ values are compared with corresponding NOR. C: Ex vivo choroidal explants were performed to assess angiogenic propensity. Choroidal tissues were obtained from rats under normoxia or OIR. One set of choroid tissues was cultured in normoxia for 48 hours; the other set was first placed in hypoxia $\left(5 \% 0_{2}\right)$ for 24 hours and returned to normoxia for another 48 hours. D: Under the normoxic conditions, choroidal explants from NOR rats expanded by 6.70 -fold (black bar; set as a relative baseline value of $100 \%$ to which all others are compared). The sprouting area of OIR rats (red bar) only increased by 3.07 -fold, merely $46 \%$ compared with NOR. Primed by hypoxia for 24 hours (followed by 48 hours in normoxia), choroids from NOR animals further expanded to 13.19-fold (197\%; white bar); those from OIR choroid continued to expand for 4.86-fold (73\%; gray bar). All changes shown in the histogram are relative to NOR cultured under normoxic conditions. $P$ values are compared with the NOR choroid culture in normoxia ( 48 hours). E: Retinal pigment epithelium (RPE)/choroid complexes (from P14 rats) were analyzed by real-time quantitative PCR arrays for factors indicated; fold changes in expression are relative to NOR. A decrease in numerous proangiogenic factors in OIR, including vascular endothelial growth factor (Vegf) receptor 2 [alias kinase insert domain receptor (Kdr)], angiopoietins 1 and 2 (Ang1/2), and most notably insulin-like growth factor 1 receptor (IGF1R; Igf1r), was detected. $P$ values are compared with corresponding NOR. Data are expressed as means \pm SEM (B, D, and E). $n=5$ to 7 animals per group $(\mathbf{B}) ; n=5$ (C and $\mathbf{D}) ; n=4$ animals per group $(\mathbf{E})$. ${ }^{*} P<0.05$, $* * P<0.01$, and ${ }^{* * *} P<0.001$. Scale bar $=30 \mu \mathrm{m}$ (B). Adgrb, adhesion G protein-coupled receptor B; Anpep, alanine aminopeptidase; Egf, epidermal growth factor; Fgf, fibroblast growth factor; Flt, Fms-like tyrosine kinase (alias Vegfr); Hif, hypoxia-inducible factor; Ifn, interferon; Irs, insulin receptor substrate; Lect, lectin; Nos, nitric oxide synthase; Nrp, neuropilin; Pecam, platelet endothelial cell adhesion molecule; Pdgf, platelet-derived growth factor; Tgfb, transforming growth factor $\beta$; Tgfbr, TGF- $\beta$ receptor; Tnf, tumor necrosis factor. 
A
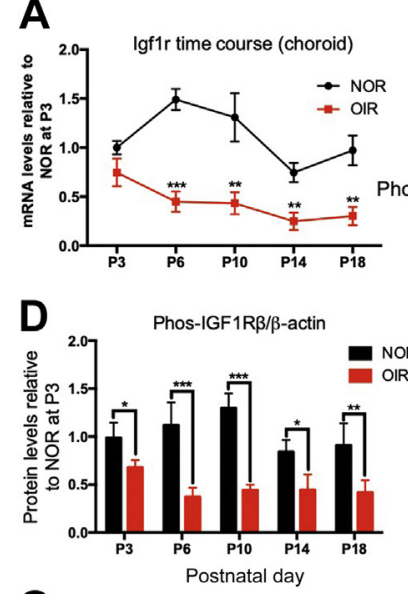

G

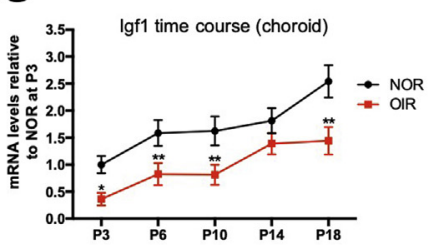

B
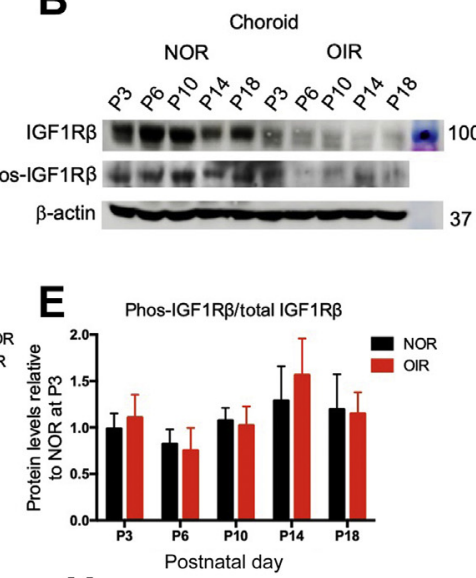

$\mathrm{H}$

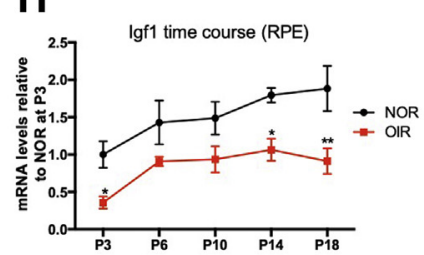

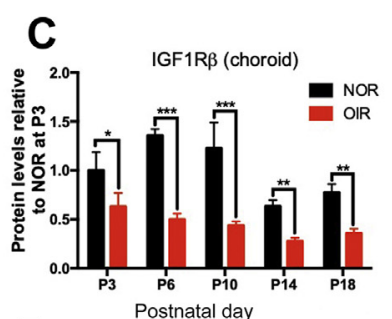

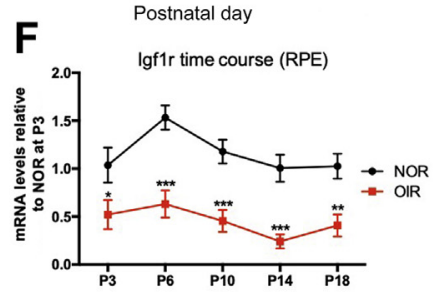

\section{I}

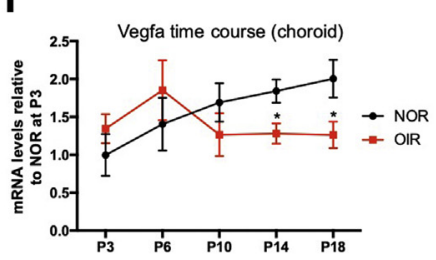

J

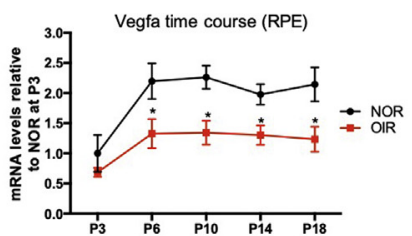

Figure 2 Down-regulation of insulin-like growth factor 1 (Igf1), Igf1 receptor (Igf1r), and vascular endothelial growth factor receptor a in retinal pigment epithelium (RPE) and choroid of oxygen-induced retinopathy (OIR)-subjected animals. A: Developmental changes in mRNA [by real-time quantitative PCR (qPCR)] and protein of Igf1 $\mathrm{r}$ in choroid of normoxia (NOR) - and OIR-subjected rats. B: Representative Western blot analysis of Igf1r and phosphorylated (phos) Igf1r from postnatal day (P) 3 to P18. C-E: Western blot analyses presented in histogram: Igf1r protein (normalized by $\beta$-actin, indicating the amount of Igf1r; C); phos-Igf1r (normalized by $\beta$-actin, indicating the amount of phos-Igf1r; D); and phos-Igf1r/Igf1r (indicating the fraction of phosphorylated Igf1r; E) (please note that each ratio was further normalized to that of P3 NOR). F: Developmental changes in mRNA (by qPCR) of Igf1r in the RPE of NOR and OIR rats. $\mathbf{G}$ and $\mathbf{H}$ : Developmental changes in mRNA of Igf1 in choroid and RPE of NOR- and OIR-subjected rats. I and J: Developmental changes in mRNA of Vegfa in choroid and RPE of NOR- and OIR-subjected rats. Data are expressed as means \pm SEM fold change from NOR set at 1 on P3 (A and $\mathbf{C}-\mathbf{J}) . n=4$ animals per group. ${ }^{*} P<0.05,{ }^{* *} P<0.01$, and ${ }^{* *} P<0.001$ compared with corresponding NOR.

thinning mostly affects the central regions. ${ }^{3-6}$ The blunt end needle was then slowly retracted from the corneal opening to avoid reflux.

\section{Statistical Analysis}

Results are presented as means \pm SEM for all experiments. One- or two-way analysis of variance with significance $\alpha=0.05$ was used for processing data. Bonferroni post hoc analysis was used to calculate significance between groups. Two-tailed $t$-tests were used to test for significance between two means.

\section{Results}

Persistent Choroidal Involution in OIR Animals Is Associated with Widespread Down-Regulation of Angiogenic Factors, including Igf1r

Using an established OIR model in rats by alternating exposure of pups to $50 \%$ and $10 \% \mathrm{O}_{2}$ every 24 hours from P0 to P14 (Figure 1A), choroidal thickness was measured at the end of the oxygen exposure and beyond. Reduced choroidal thickness was confirmed in OIR-subjected animals and was sustained into adult age (Figure 1B), despite evidence for in situ hypoxia. ${ }^{7}$ Choroids from rats subjected to OIR exhibited limited ability to sprout in Matrigel, even on ex vivo exposure to hypoxia; hence, choroids from rats subjected to OIR displayed a reduced hypoxia-induced angiogenic propensity (Figure 1, C and D).

To determine possible mediators implicated in curtailing choroidal vascular repair during OIR, RPE/choroid complex isolated from OIR and control animals at P14 was assayed for an array of factors that affect angiogenesis (Figure 1E). PCR analysis showed a substantial increase in some inflammatory mediators, such as IL- 6 , tumor necrosis factor- $\alpha$, and IL- $1 \beta$ in $\mathrm{RPE} /$ choroid of OIR-exposed subjects, as previously reported, ${ }^{7}$ along with a widespread down-regulation of important vasosupportive factors, including angiopoietins 1 and 2, kinase insert domain receptor (alias Vegf receptor 2), platelet-derived growth factor A, and pronounced Igf1r (Figure 1E).

Because Igf1r is a prominent permissive angiogenic factor, ${ }^{32}$ a detailed age-dependent expression analysis of $I g f l r$ and its ligand Igfl was conducted in choroid and RPE on exposure to supplemental oxygen. Experiments revealed sustained depression of both Igflr and Igfl in OIR (Figure 2, A-H). In addition, the decrease in total Igf1r protein among OIR subjects was not compensated by activation (ie, phosphorylation) of the remaining Igf1r. The developmental profile of phosphorylated 
A

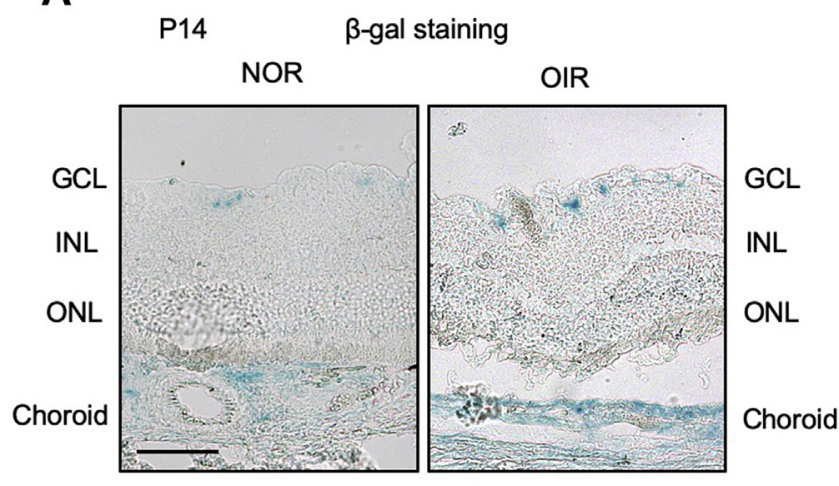

B
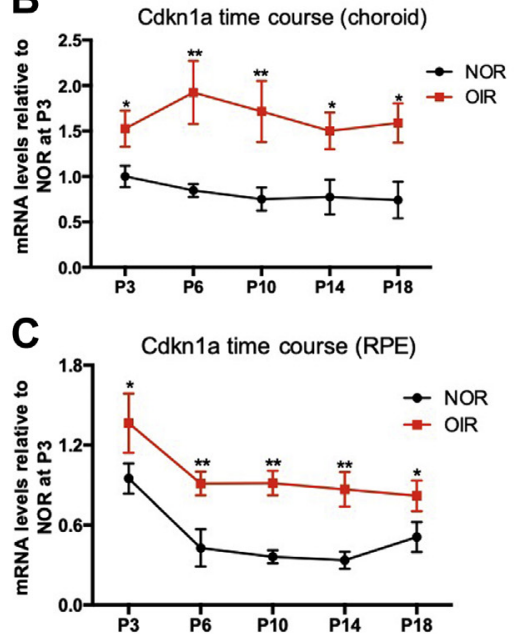

D

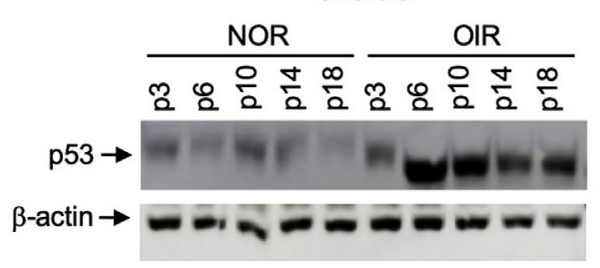

E $\quad$ 53 protein levels (choroid)

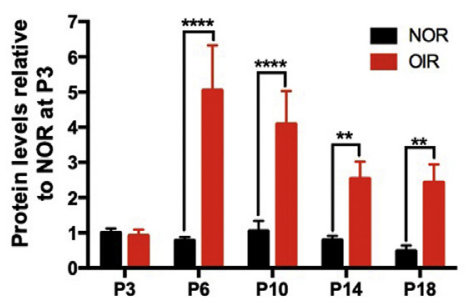

$\mathbf{F}$

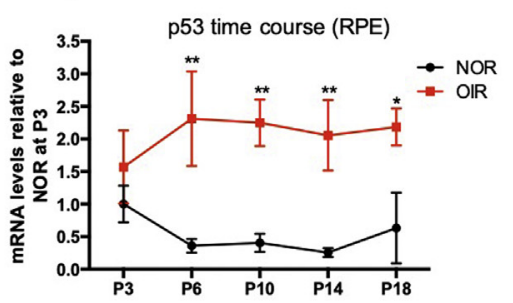

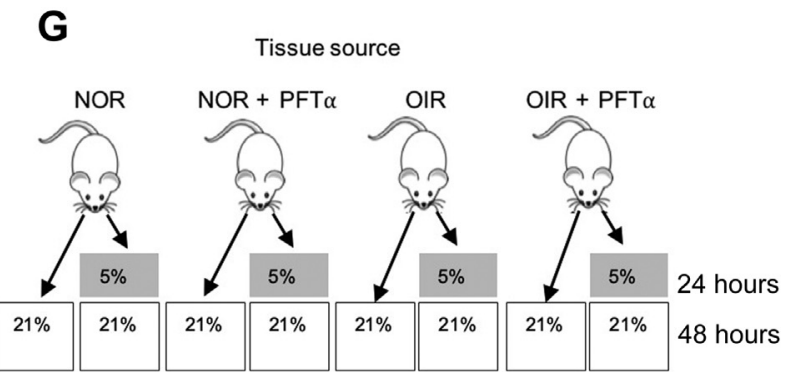

Culture environments of choroidal explants

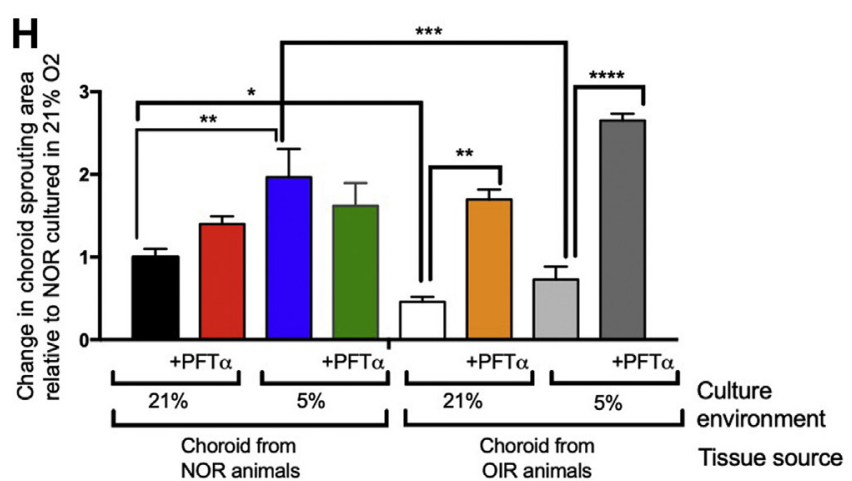

Figure 3 Senescence profile in choroid and retinal pigment epithelium (RPE) of normoxia (NOR) - and oxygen-induced retinopathy (OIR)-subjected rats. A: Representative senescence $\beta$-galactosidase ( $\beta$-gal) staining shows robust subretinal signal in RPE and choroid of OIR-subjected rats at postnatal day (P) 14. B and C: Developmental profile of cyclin-dependent kinase inhibitor 1 (Cdkn1a; p21) mRNA in choroid and RPE of NOR- and OIR-subjected rats. D-F: Time course of p53 protein expression profile in choroid and mRNA in RPE of NOR- and OIR-subjected animals. G: Ex vivo choroidal explants were performed to assess vasoproliferative ability. Choroidal tissues were collected from NOR- and OIR-subjected rats treated with pifithrin- $\alpha$ (PFT $\alpha$ ) or left untreated. One set of choroid tissues was cultured in normoxia for 48 hours, and another set was first placed in hypoxia $\left(5 \% \mathrm{O}_{2}\right)$ for 24 hours and returned to normoxia for an additional 48 hours. H: Changes in choroidal sprouting area are represented as relative to those of NOR-raised rats cultured in $21 \% \mathrm{O}_{2}$. Data are expressed as means \pm SEM (B, C, E, and $\mathbf{F}$, fold change relative to NOR set at 1 on $\mathbf{P} 3$, and $\mathbf{H}$, relative change). $n=3$ independent samples $(\mathbf{A}) ; n=4$ animals per group $(\mathbf{B}, \mathbf{C}, \mathbf{E}$, and $\mathbf{F})$; $n=5$ experiments $(\mathbf{H}) ;{ }^{*} P<0.05,{ }^{* *} P<0.01,{ }^{* * *} P<0.001$, and ${ }^{* * *} P<0.0001$ compared with corresponding NOR or as for indicated comparisons. Scale bar $=20 \mu \mathrm{m}(\mathbf{A}) . \mathrm{GCL}$, ganglion cell layer; $\mathrm{INL}$, inner nuclear layer; $0 \mathrm{NL}$, outer nuclear layer.

Igf1r (Figure 2D) was parallel to that of total Igf1r (Figure 2C); the phosphorylated Igf1r/Igf1r ratio in the OIR group did not differ at different ages (Figure 2E). Together, these data suggested that the decrease in Igf1r activity in OIR rats is almost entirely due to reduced protein expression, rather than receptor activation. Because activation of Igf1r is a prerequisite for induction (among others) of Vegfa, ${ }^{33,34}$ Vegfa expression was measured; it was significantly lower in choroid and RPE of OIR-subjected rats (Figure 2, I and J). Corresponding immunofluorescence microscopy analysis confirmed colocalization of Igflr in choroidal endothelial cells, choroidal pericytes, and RPE cells from control animals, whereas Igf1r immunofluorescence was hardly detectable in samples from OIR-subjected rats (Supplemental Figure S2, A and B). 

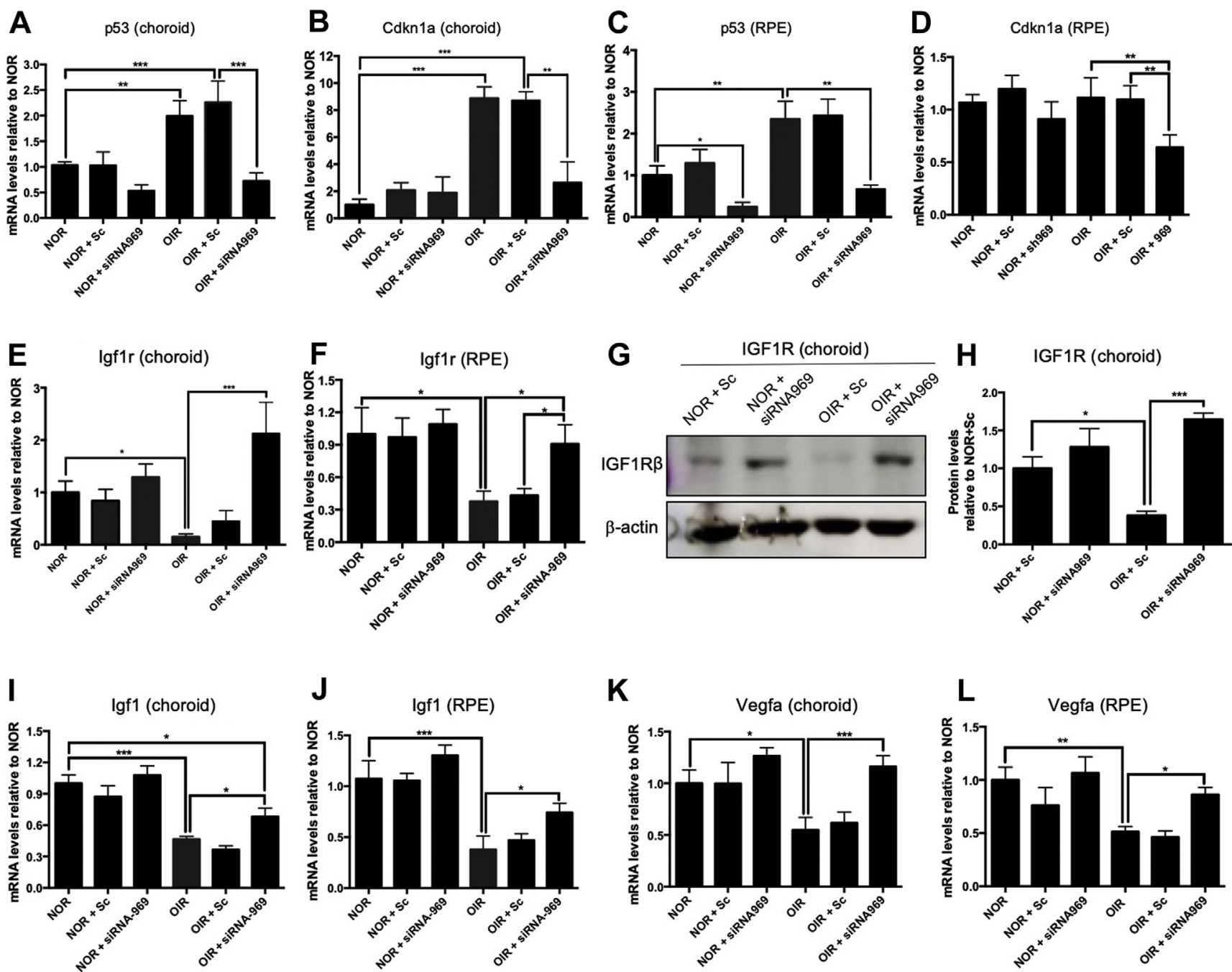

M P18
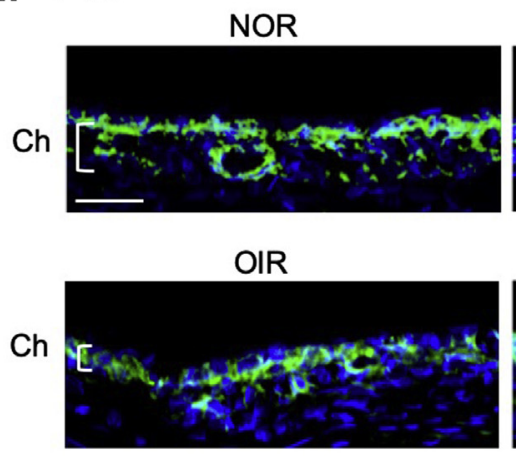

NOR+siRNA969

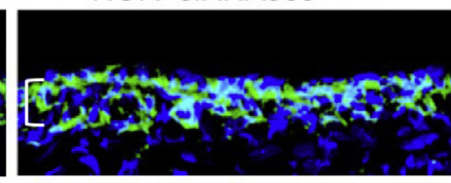

OIR+siRNA969

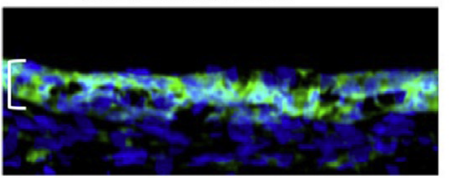

$\mathbf{N}$

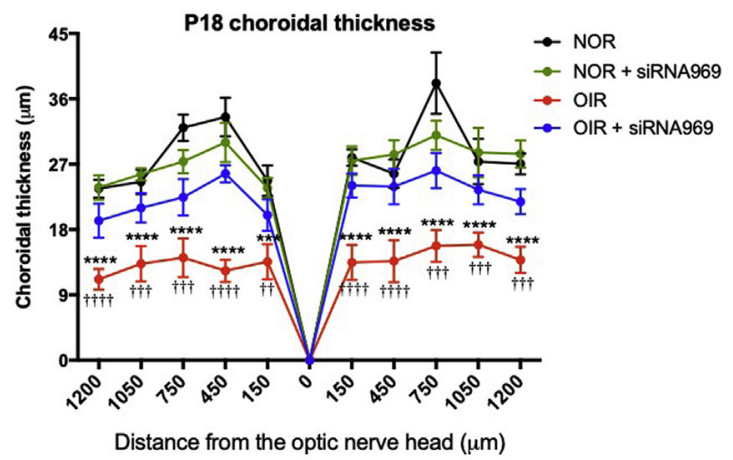

Figure 4 The p53 knockdown rescues insulin-like growth factor 1 receptor (Igf1r) [and vascular endothelial growth factor a (Vegfa)] expression and normalizes choroidal thickness. A-F and I-L: p53, cyclin-dependent kinase inhibitor 1 (Cdkn1a), Igf1r, Igf1, and Vegfa mRNA expression at postnatal day (P) 18 in central choroid and retinal pigment epithelium (RPE) of normoxia (NOR)- (set as arbitrary value of 1) and oxygen-induced retinopathy (OIR)-subjected animals treated with scrambled siRNA or (effective) siRNA969 that targets p53. Values fold change relative to N0R. G and H: Igf1r Western blot analysis and histogram quantification in choroid of NOR- (set as arbitrary value of 1) and OIR-subjected animals treated with scrambled siRNA or (effective) siRNA969. Values are fold change relative to NOR (set as 1). M: Representative immunofluorescence of choroid stained with lectin (green; vasculature) and DAPI (blue; cell nuclei). The brackets outline choroidal thickness. $\mathbf{N}$ : Choroid thickness quantification in spider graph relative to distance from optic nerve (position 0 ). Values are given of choroid thickness (in $\mu \mathrm{m})$. Data are expressed as means \pm SEM ( $\mathbf{A}-\mathbf{F}, \mathbf{H}-\mathbf{L}$, and $\mathbf{N}) . n=4$ animals per group $(\mathbf{A}-\mathbf{F}$ and $\mathbf{I}-\mathbf{L}) ; n=3$ to 5 (G and $\mathbf{H}$, experiments per group, and $\mathbf{N}$, animals per group). ${ }^{*} P<0.05,{ }^{*} P<0.01,{ }^{*} * *<0.001$, and ${ }^{* * * *} P<0.0001$ compared with corresponding NOR or as for indicated comparisons; ${ }^{\dagger \dagger} P<0.01,{ }^{\dagger \dagger \dagger} P<0.001$, and ${ }^{\dagger \dagger \dagger} P<0.0001$ compared with corresponding 0IR rats treated with siRNA969. Scale bar $=30 \mu \mathrm{m}$ (M). Ch, choroid; Sc, scrambled. 
A
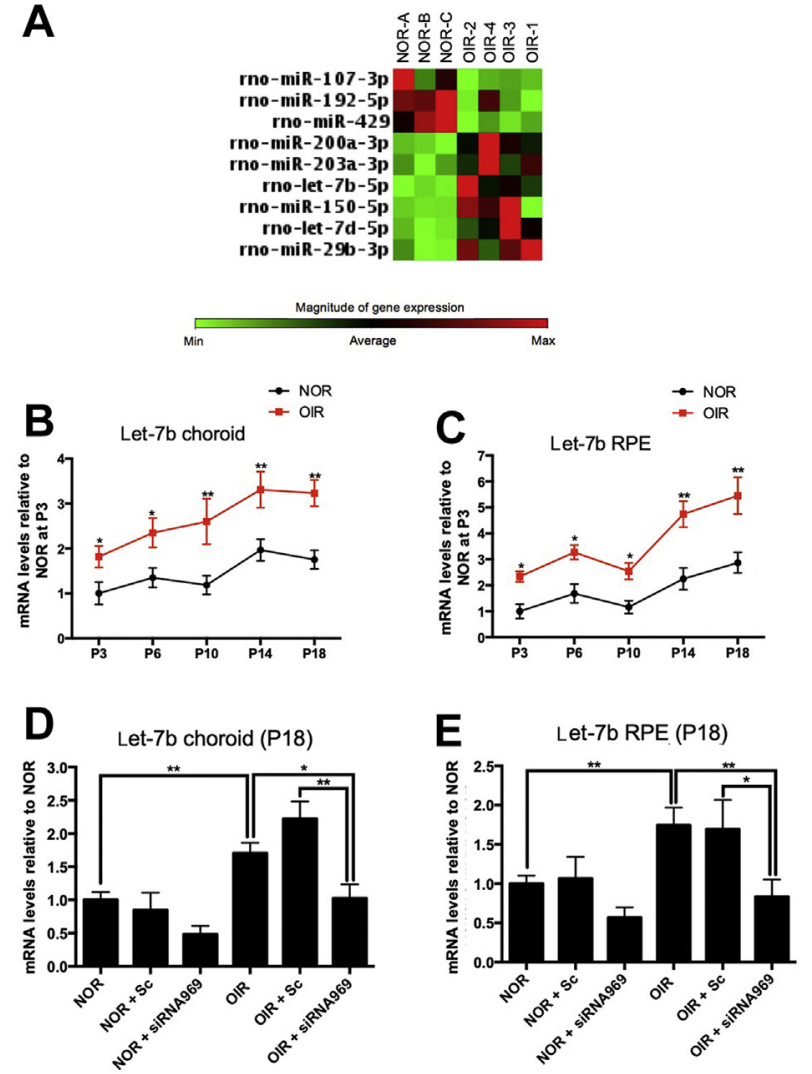

Figure 5 Relevant vasoproliferation-implicated miRNA profiling in choroid/retinal pigment epithelium (RPE) complex of animals subjected to oxygen-induced retinopathy (OIR). A: A heat map of most significantly altered miRNA expression in choroid/RPE complex of rats subjected to OIR relative to normoxia (NOR). Of 84 miRNAs that were assessed, only 8 showed statistically significant changes (increase or decrease). B and C: Time-course expression profile of let-7b in choroid and RPE of NOR- and OIR-subjected rats. Values are fold change from NOR (set at 1 ) on postnatal day (P) 3. D and E: Let-7b miRNA expression at P18 in choroid and RPE of NOR- (set as arbitrary value of 1 ) and OIR-subjected animals treated with scrambled siRNA or (effective) siRNA969 targeting p53. Values are fold change relative to NOR (set as 1). Data are expressed as means \pm SEM (B-E). $n=3$ to 4 experiments (A); $n=4$ animals per group (B and $\mathbf{C}$ ); $n=3$ to 6 experiments per group (D and $\mathbf{E}) .{ }^{*} P<0.05,{ }^{*} P P<0.01$ compared with corresponding NOR or as for indicated comparisons. Max, maximum; Min, minimum; rno, Rattus norvegicus (common rat); Sc, scrambled.

Evidence for Senescence and Up-Regulation of Tumor Suppressor p53 in Choroid and RPE of OIR-Subjected Animals

OIR is known to elicit oxidative stress, ${ }^{35}$ which affects cell replication, ${ }^{36,37}$ through effects on a broad range of gene expression. ${ }^{38}$ Increased oxidant stress in choroid and RPE in OIR witnessed soon (at P3) after oxygen exposure was confirmed. As anticipated, major reactive oxygen species generators Nox1, Nox2, and Nox4 (but not significantly Nox5) were consistently up-regulated in choroid and RPE from rats subjected to OIR (Supplemental Figure S3, A-D and H-K); coherently, major antioxidant $S O D 2$ and, to a lesser extent, SOD1 were down-regulated (Supplemental Figure S3, E, F, L, and $\mathrm{M}$ ), and expression of major oxidative stress-activated nuclear factor (erythroid-derived 2)-like $2(\mathrm{Nfe} 2 l 2)^{39}$ was augmented (Supplemental Figure S3, G and N).

Although hyperoxia and especially oxidative stress are important triggers of senescence, ${ }^{10}$ which can explain the inability of the choroid to revascularize (Figure 1, B-D), replicative cell senescence may also be contributed by local tissue hypoxia and nutrient deprivation, as seen in ischemia associated with choroidal thinning. ${ }^{7}$ Together with a decrease in growth factors, most prominently the critical Igf1r (Figure 1E), senescence-associated $\beta$-galactosidase and Cdknla (a prominent cell cycle arrest target) were assayed; and they were found to be robustly increased in choroid and RPE after OIR (Figure 3, A-C). These indicators of senescence were further supported by up-regulation of senescenceassociated tumor suppressor p53, a pivotal regulator of cell fate (involving cell cycle, apoptosis, and senescence), ${ }^{40,41}$ which, of relevance, also controls transcription of Cdknla. ${ }^{42}$ More important, p53 expression was significantly elevated from P6 and beyond oxygen exposure in choroid and RPE of OIR-subjected animals (Figure 3, D-F).

Decreased Angiogenic Ability in Choroid of OIRSubjected Animals Is Secondary to p53-Mediated Down-Regulation of Igf1r

To examine the antiangiogenic effect of p53, it was first shown that PFT $\alpha$ (a p53 inhibitor) abrogated OIR-induced increase in p53 and Cdkn1a in choroid and RPE (Supplemental Figure S4, A-C). This was associated with restoration of choroidal sprouting in response to hypoxia: PFT $\alpha$-treated hypoxia-primed OIR choriocapillary grew more robustly than untreated tissue (Figure 3, G and $\mathrm{H}$ ). Although p53 can also direct cell fate toward apoptosis, ${ }^{43}$ there were no apparent apoptotic signals (notably, terminal deoxynucleotidyl transferase-mediated dUTP nick end labeling-positive cells) in the choroid and RPE of OIRsubjected rats (Supplemental Figure S4D).

The antiangiogenic role of $\mathrm{p} 53$ was further ascertained by knocking down its expression. Of three siRNAs designed, siRNA969 was most effective in knocking down p53 in vitro (Supplemental Figure S5A); subretinal injection of siRNA969 (5 ng/mL; based on dose-response) was also

Table 4 miRNA Fold Changes

\begin{tabular}{lll}
\hline Mature identifier & Fold change & $P$ value \\
\hline rno-miR-192-5p & 0.1863 & 0.0254 \\
rno-miR-107-3p & 0.2804 & 0.0444 \\
rno-miR-429 & 0.3175 & 0.0019 \\
rno-miR-150-5p & 2.4114 & 0.0801 \\
rno-miR-203a-3p & 2.7856 & 0.0460 \\
rno-let-7d-5p & 3.2522 & 0.0318 \\
rno-miR-29b-3p & 3.3988 & 0.0185 \\
rno-let-7b-5p & 3.5211 & 0.0196 \\
rno-miR-200a-3p & 3.62 & 0.0151 \\
\hline
\end{tabular}

rno, Rattus norvegicus (common rat). 
A

Rat IGF1R ENST00000268035.6 3' UTR length: 7111

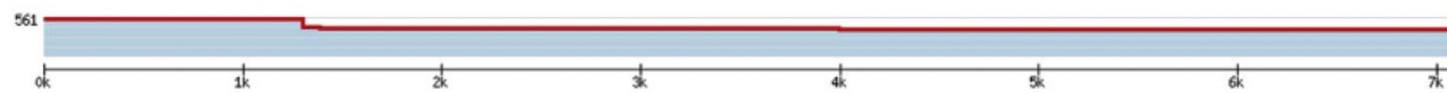

Site 1: 8 mer (2636-2643)

Site 2: 7 mer (6674-6680)

B
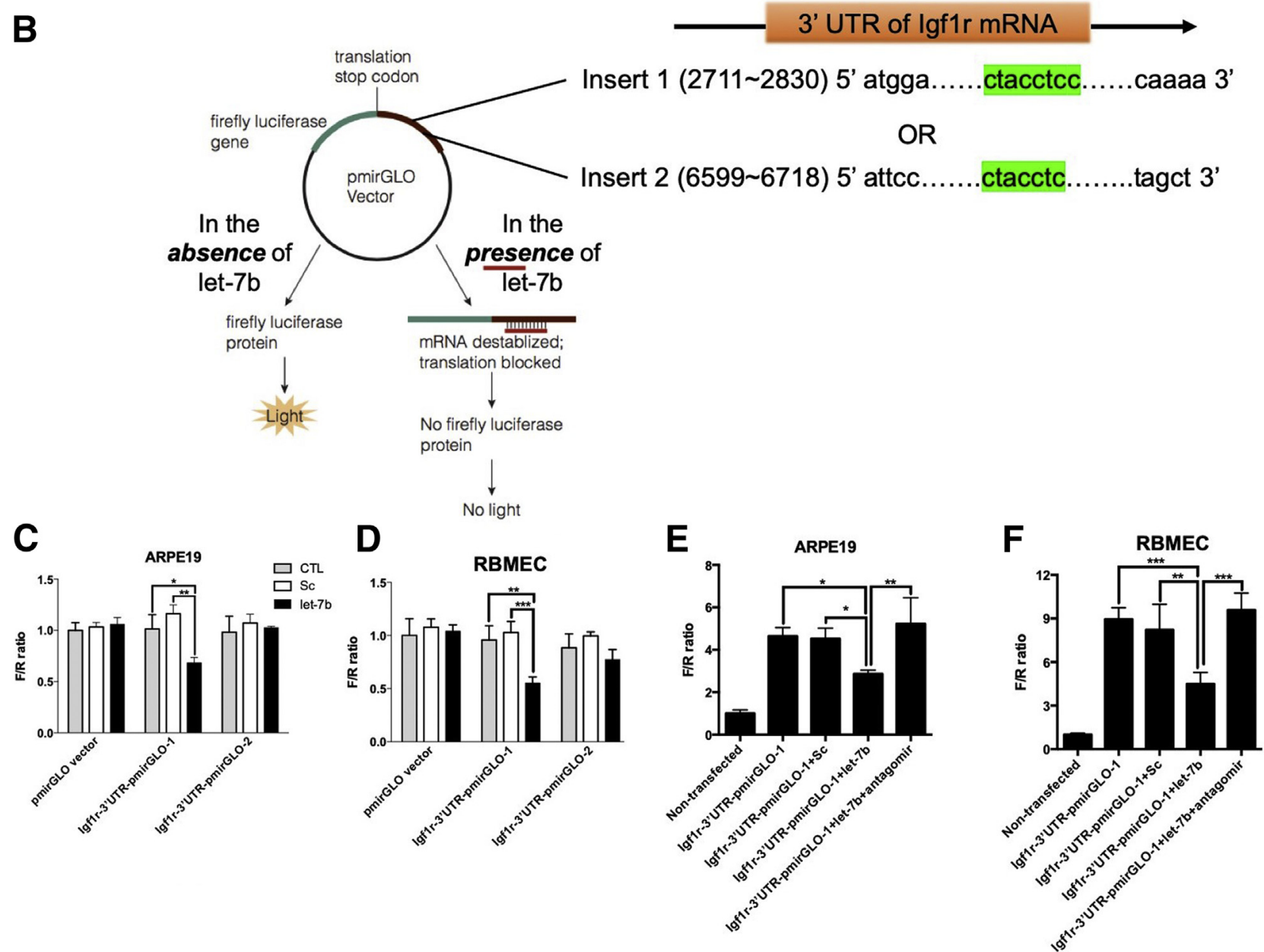

Figure 6 Dual-luciferase reporter assay confirms a let-7b target site at the $3^{\prime}$-untranslated region ( $3^{\prime}$ UTR) of insulin-like growth factor 1 receptor (Igf1r) mRNA. A: In silico prediction of miRNA targets, based on TargetScan version 6.2, reveals two potential let-7b target sites at the 3'UTR of Igf1r mRNA: an 8-mer at 2636 to 2643 (site 1) and a 7-mer at 6674 to 6680 (site 2) (yellow highlights). B: The constitution of the reporter plasmid: a fragment of Igf1r $3^{\prime} U$ UTR that contains one of the two predicted target sites was cloned downstream of the firefly luciferase gene. Both the firefly luciferase gene and the $3^{\prime} U T R$ fragment are transcribed into mRNA. In the presence of let-7b and a genuine let- $7 \mathrm{~b}$ target site, the mRNA is destabilized, or its translation is blocked, leading to a decrease in luciferase signal. The green highlighting shows the inserted sequences (1 and 2). C and D: In both ARPE19 and rat brain microvascular endothelial cells (RBMECs), cotransfection with let-7b mimics (at $20 \mathrm{nmol} / \mathrm{L}$ ) abrogated luciferase signals in those with plasmids containing target site 1 (Igf1r-3'UTR-pmirGL01) but not site 2 (Igf1r-3'UTR-pmirGL0-2). E and F: In the presence of anti-let-7b antagomir, the firefly luciferase signal was restored in cells that received Igf1r-3'UTR-pmirGL0-1 and let-7b. Values in histograms are of firefly OD/Renilla OD (F/R) ratio (see Materials and Methods). Data are expressed as means \pm SEM $(\mathbf{C}-\mathbf{F}) . n=3$ separate experiments $(\mathbf{C}-\mathbf{F}) .{ }^{*} P<0.05,{ }^{*} P<0.01$, and ${ }^{*} * *<0.001$ for indicated comparisons. CTL, control; SC, scrambled.

effective in decreasing $p 53$ expression and rescued $I g f l r$ expression (Supplemental Figure S5, B and C). More important, siRNA969 $(5 \mathrm{ng} / \mathrm{mL})$ injected subretinally (at P14) into eyes of animals subjected to OIR efficiently decreased p53 and Cdknla mRNA levels at P18 (Figure 4, A-D) and rescued Igflr, Igfl, and Vegfa expression in choroid and RPE (Figure 4, E-L); accordingly, siRNA969 treatment rescued central choroidal thickness in OIR subjects (Figure 4, M and N), along with an increase in proliferating cell nuclear antigen reactivity, indicative of cell proliferation in choroid (Supplemental Figure 5D). 


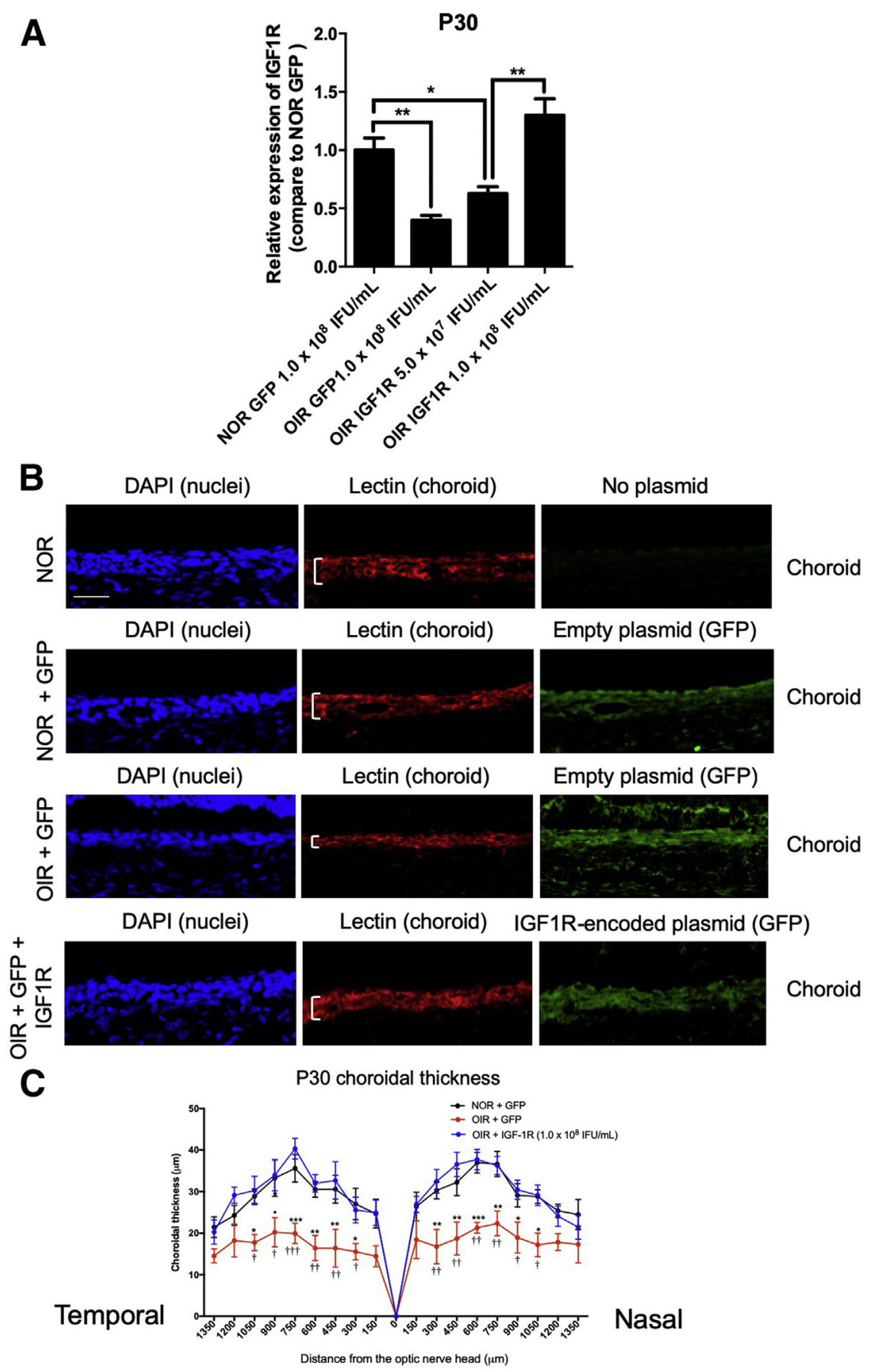

Figure 7 Subretinal restoration of insulin-like growth factor 1 receptor (Igf1r) expression rescues choroidal thickness. A: A pilot experiment was performed to identify effective dose of IGF1Rencoded lentivirus to renormalize Igf1r expression in the subretina; lentivirus encoding green fluorescent protein (GFP) was used as a control. Animals were injected subretinally at postnatal day (P) 14 and tissues were collected at P30. Lentivirus encoding IGF1R at $10^{8}$ infection units (IFU) $/ \mathrm{mL}$ (but not a lower dose) effectively normalized Igf1r expression in retinal pigment epithelium/choroid complex, comparable to that observed in normoxia (NOR). Values are of Igf1r fold change in mRNA relative to NOR. B: Representative images of central choroid (approximately $450 \mu \mathrm{m}$ away from the optic nerve) are shown. Blue, red, and green signals represent nuclei (DAPI), choroid (lectin), and GFP (or IGF1R-GFP), respectively. The brackets outline choroidal thickness. C: Choroid thickness (the spider graph) assessed from the optic nerve to nasal and temporal periphery in groups indicated. Data are expressed as means \pm SEM (A and $\mathbf{C}) . n$ $=5$ separate experiments $(\mathbf{A}$ and $\mathbf{C})$. ${ }^{*} P<0.05$, $* * P<0.01$, and $* * * P<0.001$ compared with corresponding NOR or as for indicated comparisons; ${ }^{\dagger} P<0.05,{ }^{\dagger \dagger} P<0.01$, and ${ }^{{ }^{\dagger \dagger}} P<0.001$ compared with corresponding oxygen-induced retinopathy (OIR) animals injected with IGF1Rencoded lentivirus. Scale bar $=50 \mu \mathrm{m}$ (B).

\section{p53 Down-Regulates Igf1r Expression by Inducing Let-7b miRNA}

To determine how p53 governs the expression of Igf1r in OIR and given that numerous factors are concomitantly affected (Figure 1E), the effect of OIR in altering expression of miRNAs and the role of p53 in governing these miRNAs were evaluated. ${ }^{19,22,44}$ Using an 84 miRNA PCR array to profile miRNAs involved in vasoproliferation, eight miRNAs (namely, miR- 107-3p, miR-192-5p, miR-429, let-7b-5p, let-7d-5p, miR200a-3p, miR-203a-3p, and miR-29b-3p) exhibited statistically significant changes by OIR (Figure 5A and Table 4). Of interest, only let-7b-5p (referred as let-7b hereafter), recently implicated in the inhibition of choroidal neovascularization in diabetic retinopathy, ${ }^{45}$ was consistently found to be elevated in a similar way as p53 (from P3 to P18) in choroid and RPE of OIR-exposed rats (Figure 5, B and C), suggesting a potential regulatory interaction between p53 and let-7b; the other 
miRNAs showed an irregular and an inconsistent pattern relative to 553 in RPE or in choroid throughout the period analyzed from P3 to P18 (compared with Supplemental Figure S6). Moreover, miRNA profiling in OIR in rats using nextgeneration sequencing revealed let- $7 \mathrm{~b}$ as one of the most expressed miRNAs in choroid. ${ }^{46}$ In line with these observations, expression of the p53-regulated miRNA binding protein Lin $28,{ }^{15}$ which suppresses let-7b function, ${ }^{47}$ was found to be decreased in OIR (Supplemental Figure S7). Although other potential p53 downstream pathways were considered, ${ }^{19}$ these did not seem to be relevant herein, as was the case for VEGF receptor 1 and hypoxia-inducible factor- $1 \alpha$, which were unaltered by OIR and/or PFT $\alpha$ (Supplemental Figure S7). Thus, because let-7b is an important regulator of Igf1 $\mathrm{r}$ in cancer cells, ${ }^{48,49}$ itself controlled by p53 (Figure 4), the link between p53 and let-7b was further corroborated by p53 knock-down (using siRNA969), which normalized let-7b expression in choroid and RPE of animals subjected to OIR at P14 (Figure 5, D and E).

\section{Confirming the Igf1r Transcript Is a True Target of Let-7b}

On the basis of evidence that let-7 family miRNAs target the Igf1 pathway in cancer and diabetes, ${ }^{23,50}$ this evidence was confirmed in RPE and neurovascular endothelial cells. In silico prediction of potential miRNA targets using TargetScan version 6.2 revealed two potential let-7b target sites on the 3'UTR of Igflr mRNA: an 8-mer at 2636 to 2643 (site 1) and a 7 -mer at 6674 to 6680 (site 2) (Figure 6A). These sequences were inserted downstream of luciferase firefly gene into a pmirGLO vector (Figure 6B); individual constructs and synthetic let-7b mimic (Syn-rno/hsa-let-7b-5p, effective at 20 nmol/L) (Supplemental Figure S8) were then transfected into ARPE-19 and neurovascular endothelial cells. In the presence of let-7b mimics, luciferase activity (measured as the firefly $\mathrm{OD} /$ Renilla OD ratio) diminished in the groups receiving the construct that contains target site 1 (2636 to 2643) but not site 2 (6674 to 6680), indicating the former as the valid target site of let-7b (Figure 6, C and D); the scrambled sequence did not alter Igflr expression. In addition, cotransfection of let-7b mimic with its antagomir preserved luciferase activity (Figure 6, E and F). These data collectively indicate that the Igfl $r$ mRNA is targeted by let-7b.

\section{Restoration of Igf1r Expression Rescues Choroidal Thickness after OIR}

As choroidal thickness remains sustained beyond the high oxygen exposure, it was determined if restoring the levels of Igf1r in the subretina could rescue choroidal thickness. Because the amount of let- $7 \mathrm{~b}$ antagomirs needed for in vivo experiments was calculated to be high and possibly result in undesired effects, Igf1r was subretinally restored in OIR and it was determined if the latter would reinstate normal choroidal thickness. Lentiviral particles encoded with green fluorescent protein (negative control) and/or Igf1r were injected into the subretinal space at low $\left[5.0 \times 10^{7}\right.$ infection units $(\mathrm{IFU}) / \mathrm{mL}]$ or high $\left(1.0 \times 10^{8} \mathrm{IFU} / \mathrm{mL}\right)$ doses in OIR rats at P14. Two weeks later (at P30), the expression level of Igf1r was evaluated by real-time quantitative PCR and immunohistochemistry (Figure 7A and Supplemental Figure S9). Choroidal thickness was also assessed. The higher dose of lentivirus encoded with Igf1r increased receptor expression in the subretina (Figure 7B) and rescued choroid thickness (Figure 7, B and C). Hence, Igf1r exerts a significant role in reinstating choroidal vascular integrity. Altogether, inhibiting p53/let-7b pathway and/or restoring the levels of Igf1r in the subretina can avert choroidal involution and reestablish its integrity (Supplemental Figure S10).

\section{Discussion}

The inner retina has long been considered to be the primary region affected in human ROP and animal models. ${ }^{2}$ Lately, dysfunctions of the outer retina and subretina, composed of photoreceptors, RPE, and choroid, have been detected in a significant number of older children formerly afflicted with ROP. ${ }^{8,51,52}$ Choroidal involution was confirmed in the OIR model ${ }^{6}$ and resulted in hypoxic ischemia, which, in turn, compromised photoreceptor integrity. ${ }^{7}$ Prompt revascularization is considered a desirable physiological response to ischemia to prevent long-term damage. ${ }^{53}$ Strikingly and unlike the retinal (and brain) vasculature, ${ }^{2}$ choroidal revascularization is negligible and involution is sustained long after OIR/ROP affliction. ${ }^{3-6}$ IL-1 $\beta$ contributes significantly to choroidal thinning, and early anti-IL$1 \beta$ treatment (during OIR induction) prevents choroidal involution $^{7}$; however, late anti-IL-1 $\beta$ intervention (starting at P60) fails to rescue an involuted choroid. ${ }^{7}$ In the present study, for the first time, molecular mechanisms underlying the curtailed ability of the choroid to revascularize after OIR/ROP-induced choroidal thinning were reported. These findings reveal evidence for oxidative and possibly hypoxicischemic stress-related replicative senescence in OIR associated with up-regulation of cell fate-relevant p53 and downstream let-7b miRNA in the subretina, resulting in suppression of major permissive growth factor Igf1r (and its ligand Igf1); p53 inhibition or gene silencing enhanced choroidal vasoproliferation as it depressed let-7b expression, normalized Igf1r, and restored choroidal thickness, as observed with Igflr gene rescue.

A salient feature of this study applies to the role of p53 in ROP/OIR, which has never been addressed before, particularly in the context of the subretina. Herein, we imply involvement of p53 in sustained choroidal thinning and inability to recover choroidal integrity. It was first shown that an important trigger of p53 expression (specifically, oxidative stress) was detected in the subretina, as witnessed by early up-regulation of NADPH oxidase (superoxide- 
producing enzyme) transcripts, suppression of SOD, and an increase in oxidative stress-response transcription factor $\mathrm{Nfe} 212$ [alias nuclear respiratory factor 2 (Nrf2)]. ${ }^{54}$ Moreover, NOXs and Nrf2 remained increased 3 to 6 days after supplemental oxygen exposure. Oxidative stress stabilizes and activates p53, ${ }^{10,55}$ along with Cdkn1a. ${ }^{56}$ Yet, one cannot exclude a role for choroid/RPE hypoxic ischemia in p53-related senescence as a result of choroidal thinning. Second, pharmacologic inhibition (PFT $\alpha$ ) and gene knockdown of p53 restored choroidal endothelium's ability to sprout and increased choroid thickness and revascularization (indicated by increased proliferating cell nuclear antigen). These findings are consistent with effects of Nutlin-3, which is p53 pathway dependent, in inhibiting hind limb and intravitreal retinal neovascularization. ${ }^{57}$ Third, oxidative stress preceded increased p53 expression as the two are tightly linked to cellular senescence ${ }^{16}$; hypoxic ischemia may also participate in this cell senescence after P6.

The p53 lies unequivocally at the crossroads of cell fate, primarily homeostatic repair and senescence. ${ }^{58}$ Mechanisms for distinct roles of p53 on diverse cell fates appear to imply differences in interactions of the various isoforms of $\mathrm{p} 53$ with distinct target proteins involved in cell fate ${ }^{59}$ and coinduction of epigenetic regulatory mechanisms in response to original stressors. ${ }^{60}$ In line with its role in senescence, p53 can interfere with actions of various growth-promoting factors, ${ }^{61}$ including induction of the Igf1r pathway. ${ }^{62}$ It is also suggested that transient activation of p53 in response to a low dosage of oxidative stress favors senescence, whereas persistent activation of p53 during massive oxidative stress can result in cell death ${ }^{63}$; yet notably, apoptosis was not detected in the choroid of OIRsubjected animals.

Of interest, the choroid and RPE exhibited similar patterns of $p 53$, Cdknla, let-7b, Igflr, and Igfl expression in animals subjected to OIR. Moreover, these factors also seem to be similarly regulated by p53 in choroid and RPE. In this context, both replicative and post-mitotic cells can be targeted by oxidative stress and hypoxia-ischemia, as seen in OIR, resulting in an endoplasmic reticulum stress that can be a gateway to a senescence phenotype ${ }^{64,65}$ Hence, given that the RPE contributes to choroidal integrity, the impact on the choroid appears dual, such that replicative senescence would directly curtail choroid thickness, whereas nonreplicative senescence in RPE could additionally indirectly affect choroid function through paracrine effects. ${ }^{12,13}$ The senescent phenotype in RPE may thus amplify the inability of the choroidal endothelium to proliferate, compared with the revascularization that takes place in the retinal vasculature in OIR/ROP. In this context, a role for antiinflammatory cytokines, such as IL-10 and substance P, may also participate. ${ }^{66,67}$

Recent efforts have been made to identify crucial miRNA-target pairs that regulate retinal neovascularization. ${ }^{68,69}$ Among them, the let-7 family of miRNAs has been detected in the plasma of patients with wet age-related macular degeneration ${ }^{70}$ and is down-regulated in retinal endothelium of streptozotocin-induced diabetic rats. ${ }^{71}$ miRNA profiling in choroid of OIR-subjected rats highlights the abundance of let- $7 \mathrm{~b},{ }^{46}$ consistent with current observations. In addition, the luciferase reporter system confirms the let-7b target site on the $3^{\prime}$ UTR of Igflr mRNA (2636 to 2643) in cultured RPE and neurovascular endothelial cells.

Although the interaction between let-7 and IGF1R has been described in cancer and diabetes, ${ }^{23,50}$ the p53-let-7bIgf1r regulatory axis is new to retinopathies. It has previously been shown that p53 does not directly bind to Igflr promoter, ${ }^{72}$ but rather regulates the Igf1 pathway by modulating antiproliferative miRNAs. ${ }^{19}$ The let-7 family stands out particularly intertwined with the Igf1 axis. ${ }^{23,48,73}$ Biogenesis of the let-7 family is enhanced by p53, which represses Lin28, an RNA binding protein that precludes let7 maturation and action. ${ }^{22,74,75}$ Consistent with increased p53 and let-7b in OIR, Lin28 was suppressed.

At first glance, down-regulation of prosurvival/angiogenic Igf1r in the subretina in OIR as the choroid thins is paradoxical, especially in ischemic retinopathy, where retinal neovascularization is driven by ischemia-hypoxia. Igf1r, which is ubiquitously expressed, is linked to elevated metabolism, ${ }^{76}$ contributes to oxidative stress generation, and affects longevity. ${ }^{77,78}$ Thus, a reduction in Igf1r, as observed herein, would reduce metabolic rate in OIR, ${ }^{79}$ and intuitively be considered beneficial. On the other hand, on IGF1 stimulation during hypoxia, ${ }^{80,81}$ RPE releases proangiogenic/prosurvival IGF1/2, IGF-binding proteins $3 / 6$ (key binding proteins for bioactive IGF1/2), and VEGFa. Conversely, low postnatal serum Igf1 values have been strongly correlated with development of $\mathrm{ROP}^{82}$; but supplementing preterm infants with IGF-1 failed to prevent ROP (clinical trial: NCT01096784), possibly because of limited Igf1r expression in the inner retina. Low Igf1r, Igf1, and Vegfa expression is also observed in the subretina of OIR subjects; a defect in RPE-derived VEGF can lead to choriocapillaris atrophy and deleterious choroidal remodeling, ${ }^{83}$ which share pathologic features with middle-aged OIR/ROP subjects (notably, choroidal degeneration, abnormalities in RPE, and photoreceptor dysfunction). ${ }^{7}$ All in all, a reduction in the expression of Igf1, Igf1r, and Vegfa, as seen in the outer retina of OIR subjects, generates an unfavorable environment for choroidal revascularization, resulting in persistent choroidal involution; decreased Igf1r signaling in the RPE may further compromise photoreceptor function. ${ }^{7,8,84}$

Collectively, this study reveals an unprecedented mechanism to explain the inability of the choroid to revascularize after OIR-induced involution. This study unveils, for the first time, the critical role of p53-let-7b-Igflr axis in orchestrating a defective angiogenic environment in the subretina, which impedes choroidal revascularization. Targeting this pathway represents a potential therapeutic avenue to rescue choroidal involution in ROP subjects and other chorioretinopathies, as in geographic atrophy. 


\section{Acknowledgments}

We thank Dr. Neeraj Agarwal (National Eye Institute, Bethesda, MD) for providing the retinal ganglion cell line; and Dr. Stanley Nattel for providing the pmirGLO DualLuciferase miRNA Target Expression Vector.

T.E.Z. and S.C. jointly designed the study and wrote the manuscript; T.E.Z. performed most experiments and data analyses; J.C.R. wrote the manuscript and provided expertise advice; M.W. analyzed data; S.O. revised the manuscript and provided expert advice; G.L., G.F., and M.D. provided expertise related to senescence, endothelial proliferation, and ischemic stress; I.L. prepared cryosection slides; H.T. performed part of the immunohistochemistry experiments; T.Z., R.R., and M.N. performed real-time quantitative PCR and Western blot analysis experiments; S.N. provided materials and expert advice on miRNA experiments; and P.H. collected and analyzed optical coherence tomography images from neonates.

\section{Supplemental Data}

Supplemental material for this article can be found at http://doi.org/10.1016/j.ajpath.2019.07.009.

\section{References}

1. Gilbert C: Retinopathy of prematurity: a global perspective of the epidemics, population of babies at risk and implications for control. Early Hum Dev 2008, 84:77-82

2. Sapieha P, Joyal JS, Rivera JC, Kermorvant-Duchemin E, Sennlaub F, Hardy P, Lachapelle P, Chemtob S: Retinopathy of prematurity: understanding ischemic retinal vasculopathies at an extreme of life. J Clin Invest 2010, 120:3022-3032

3. Wu WC, Shih CP, Wang NK, Lien R, Chen YP, Chao AN, Chen KJ, Chen TL, Hwang YS, Lai CC, Huang CY, Tsai S: Choroidal thickness in patients with a history of retinopathy of prematurity. JAMA Ophthalmol 2013, 131:1451-1458

4. Erol MK, Coban DT, Ozdemir O, Dogan B, Tunay ZO, Bulut M: Choroidal thickness in infants with retinopathy of prematurity. Retina 2016, 36:1191-1198

5. Anderson MF, Ramasamy B, Lythgoe DT, Clark D: Choroidal thickness in regressed retinopathy of prematurity. Eye (Lond) 2014, 28:1461-1468

6. Shao Z, Dorfman AL, Seshadri S, Djavari M, KermorvantDuchemin E, Sennlaub F, Blais M, Polosa A, Varma DR, Joyal JS, Lachapelle P, Hardy P, Sitaras N, Picard E, Mancini J, Sapieha P, Chemtob S: Choroidal involution is a key component of oxygeninduced retinopathy. Invest Ophthalmol Vis Sci 2011, 52:6238-6248

7. Zhou TE, Rivera JC, Bhosle VK, Lahaie I, Shao Z, Tahiri H, Zhu T, Polosa A, Dorfman A, Beaudry-Richard A, Costantino S, Lodygensky GA, Lachapelle P, Chemtob S: Choroidal involution is associated with a progressive degeneration of the outer retinal function in a model of retinopathy of prematurity: early role for IL-1beta. Am J Pathol 2016, 186:3100-3116

8. Hansen RM, Moskowitz A, Akula JD, Fulton AB: The neural retina in retinopathy of prematurity. Prog Retin Eye Res 2017, 56:32-57

9. Yu DY, Cringle SJ, Alder V, Su EN: Intraretinal oxygen distribution in the rat with graded systemic hyperoxia and hypercapnia. Invest Ophthalmol Vis Sci 1999, 40:2082-2087
10. Finkel T, Holbrook NJ: Oxidants, oxidative stress and the biology of ageing. Nature 2000, 408:239-247

11. Xie S, Wang Q, Wu H, Cogswell J, Lu L, Jhanwar-Uniyal M, Dai W: Reactive oxygen species-induced phosphorylation of p53 on serine 20 is mediated in part by polo-like kinase-3. J Biol Chem 2001, 276: 36194-36199

12. Pluquet O, Pourtier A, Abbadie C: The unfolded protein response and cellular senescence: a review in the theme: cellular mechanisms of endoplasmic reticulum stress signaling in health and disease. Am J Physiol Cell Physiol 2015, 308:C415-C425

13. Sapieha P, Mallette FA: Cellular senescence in postmitotic cells: beyond growth arrest. Trends Cell Biol 2018, 28:595-607

14. Yokoyama M, Shimizu I, Nagasawa A, Yoshida Y, Katsuumi G, Wakasugi T, Hayashi Y, Ikegami R, Suda M, Ota Y, Okada S, Fruttiger M, Kobayashi Y, Tsuchida M, Kubota Y, Minamino T: p53 plays a crucial role in endothelial dysfunction associated with hyperglycemia and ischemia. J Mol Cell Cardiol 2019, 129: 105-117

15. Boominathan L: The tumor suppressors p53, p63, and p73 are regulators of microRNA processing complex. PLoS One 2010, 5:e10615

16. Liu D, Xu Y: p53, oxidative stress, and aging. Antioxid Redox Signal 2011, 15:1669-1678

17. Fujita K, Mondal AM, Horikawa I, Nguyen GH, Kumamoto K, Sohn JJ, Bowman ED, Mathe EA, Schetter AJ, Pine SR, Ji H, Vojtesek B, Bourdon JC, Lane DP, Harris CC: p53 isoforms Delta133p53 and p53beta are endogenous regulators of replicative cellular senescence. Nat Cell Biol 2009, 11:1135-1142

18. Luo Z, Cui R, Tili E, Croce C: Friend or foe: microRNAs in the p53 network. Cancer Lett 2018, 419:96-102

19. Hermeking $\mathrm{H}$ : MicroRNAs in the p53 network: micromanagement of tumour suppression. Nat Rev Cancer 2012, 12:613-626

20. Roybal JD, Zang Y, Ahn YH, Yang Y, Gibbons DL, Baird BN, Alvarez C, Thilaganathan N, Liu DD, Saintigny P, Heymach JV, Creighton CJ, Kurie JM: miR-200 inhibits lung adenocarcinoma cell invasion and metastasis by targeting Flt1/VEGFR1. Mol Cancer Res 2011, 9:25-35

21. Panda H, Pelakh L, Chuang TD, Luo X, Bukulmez O, Chegini N: Endometrial miR-200c is altered during transformation into cancerous states and targets the expression of ZEBs, VEGFA, FLT1, IKKbeta, KLF9, and FBLN5. Reprod Sci 2012, 19:786-796

22. Lee JY, Kim HJ, Yoon NA, Lee WH, Min YJ, Ko BK, Lee BJ, Lee A, Cha HJ, Cho WJ, Park JW: Tumor suppressor p53 plays a key role in induction of both tristetraprolin and let-7 in human cancer cells. Nucleic Acids Res 2013, 41:5614-5625

23. Zhu H, Shyh-Chang N, Segre AV, Shinoda G, Shah SP, Einhorn WS, Takeuchi A, Engreitz JM, Hagan JP, Kharas MG, Urbach A, Thornton JE, Triboulet R, Gregory RI; DIAGRAM Consortium; MAGIC Investigators, Altshuler D, Daley GQ: The Lin28/let-7 axis regulates glucose metabolism. Cell 2011, 147:81-94

24. Xu H, Liu C, Zhang Y, Guo X, Liu Z, Luo Z, Chang Y, Liu S, Sun Z, Wang X: Let-7b-5p regulates proliferation and apoptosis in multiple myeloma by targeting IGF1R. Acta Biochim Biophys Sin (Shanghai) 2014, 46:965-972

25. Penn JS, Henry MM, Tolman BL: Exposure to alternating hypoxia and hyperoxia causes severe proliferative retinopathy in the newborn rat. Pediatr Res 1994, 36:724-731

26. Dorfman A, Dembinska O, Chemtob S, Lachapelle P: Early manifestations of postnatal hyperoxia on the retinal structure and function of the neonatal rat. Invest Ophthalmol Vis Sci 2008, 49: $458-466$

27. Tahiri H, Yang C, Duhamel F, Omri S, Picard E, Chemtob S, Hardy P: p75 neurotrophin receptor participates in the choroidal antiangiogenic and apoptotic effects of T-lymphocyte-derived microparticles. Invest Ophthalmol Vis Sci 2013, 54:6084-6092

28. Joyal JS, Sitaras N, Binet F, Rivera JC, Stahl A, Zaniolo K, Shao Z, Polosa A, Zhu T, Hamel D, Djavari M, Kunik D, Honore JC, Picard E, Zabeida A, Varma DR, Hickson G, Mancini J, Klagsbrun M, 
Costantino S, Beausejour C, Lachapelle P, Smith LE, Chemtob S, Sapieha P: Ischemic neurons prevent vascular regeneration of neural tissue by secreting semaphorin 3A. Blood 2011, 117:6024-6035

29. Marler KJ, Suetterlin P, Dopplapudi A, Rubikaite A, Adnan J, Maiorano NA, Lowe AS, Thompson ID, Pathania M, Bordey A, Fulga T, Van Vactor DL, Hindges R, Drescher U: BDNF promotes axon branching of retinal ganglion cells via miRNA-132 and p250GAP. J Neurosci 2014, 34:969-979

30. Han F, Huo Y, Huang CJ, Chen CL, Ye J: MicroRNA-30b promotes axon outgrowth of retinal ganglion cells by inhibiting Semaphorin3A expression. Brain Res 2015, 1611:65-73

31. Tiscornia G, Singer O, Verma IM: Production and purification of lentiviral vectors. Nat Protoc 2006, 1:241-245

32. Hellstrom A, Perruzzi C, Ju M, Engstrom E, Hard AL, Liu JL, Albertsson-Wikland K, Carlsson B, Niklasson A, Sjodell L, LeRoith D, Senger DR, Smith LE: Low IGF-I suppresses VEGFsurvival signaling in retinal endothelial cells: direct correlation with clinical retinopathy of prematurity. Proc Natl Acad Sci U S A 2001, 98:5804-5808

33. Slomiany MG, Rosenzweig SA: IGF-1-induced VEGF and IGFBP-3 secretion correlates with increased HIF-1 alpha expression and activity in retinal pigment epithelial cell line D407. Invest Ophthalmol Vis Sci 2004, 45:2838-2847

34. Fukuda R, Hirota K, Fan F, Jung YD, Ellis LM, Semenza GL: Insulin-like growth factor 1 induces hypoxia-inducible factor 1mediated vascular endothelial growth factor expression, which is dependent on MAP kinase and phosphatidylinositol 3-kinase signaling in colon cancer cells. J Biol Chem 2002, 277:38205-38211

35. Rivera JC, Dabouz R, Noueihed B, Omri S, Tahiri H, Chemtob S: Ischemic retinopathies: oxidative stress and inflammation. Oxid Med Cell Longev 2017, 2017:3940241

36. Chen Q, Fischer A, Reagan JD, Yan LJ, Ames BN: Oxidative DNA damage and senescence of human diploid fibroblast cells. Proc Natl Acad Sci U S A 1995, 92:4337-4341

37. Parrinello S, Samper E, Krtolica A, Goldstein J, Melov S, Campisi J: Oxygen sensitivity severely limits the replicative lifespan of murine fibroblasts. Nat Cell Biol 2003, 5:741-747

38. Morel Y, Barouki R: Repression of gene expression by oxidative stress. Biochem J 1999, 342 Pt 3:481-496

39. Gorrini C, Harris IS, Mak TW: Modulation of oxidative stress as an anticancer strategy. Nat Rev Drug Discov 2013, 12:931-947

40. Brown CJ, Lain S, Verma CS, Fersht AR, Lane DP: Awakening guardian angels: drugging the p53 pathway. Nat Rev Cancer 2009, 9: $862-873$

41. Graeber TG, Peterson JF, Tsai M, Monica K, Fornace AJ Jr, Giaccia AJ: Hypoxia induces accumulation of p53 protein, but activation of a G1-phase checkpoint by low-oxygen conditions is independent of p53 status. Mol Cell Biol 1994, 14:6264-6277

42. Murray-Zmijewski F, Slee EA, Lu X: A complex barcode underlies the heterogeneous response of p53 to stress. Nat Rev Mol Cell Biol 2008, 9:702-712

43. Hafner A, Bulyk ML, Jambhekar A, Lahav G: The multiple mechanisms that regulate p53 activity and cell fate. Nat Rev Mol Cell Biol 2019, 20:199-210

44. Pichiorri F, Suh SS, Rocci A, De Luca L, Taccioli C, Santhanam R, Zhou W, Benson DM Jr, Hofmainster C, Alder H, Garofalo M, Di Leva G, Volinia S, Lin HJ, Perrotti D, Kuehl M, Aqeilan RI, Palumbo A, Croce CM: Downregulation of p53-inducible microRNAs 192, 194, and 215 impairs the p53/MDM2 autoregulatory loop in multiple myeloma development. Cancer Cell 2010, 18:367-381

45. Zhou Q, Frost RJA, Anderson C, Zhao F, Ma J, Yu B, Wang S: Let-7 contributes to diabetic retinopathy but represses pathological ocular angiogenesis. Mol Cell Biol 2017, 37:e00001-17

46. Desjarlais M, Rivera JC, Lahaie I, Cagnone G, Wirt M, Omri S, Chemtob S: MicroRNA expression profile in retina and choroid in oxygen-induced retinopathy model. PLoS One 2019, 14:e0218282
47. Stefani G, Chen X, Zhao H, Slack FJ: A novel mechanism of LIN-28 regulation of let-7 microRNA expression revealed by in vivo HITSCLIP in C. elegans. RNA 2015, 21:985-996

48. Gao L, Wang X, Wang X, Zhang L, Qiang C, Chang S, Ren W, Li S, Yang Y, Tong D, Chen C, Li Z, Song T, Zhi K, Huang C: IGF-1R, a target of let-7b, mediates crosstalk between IRS-2/Akt and MAPK pathways to promote proliferation of oral squamous cell carcinoma. Oncotarget 2014, 5:2562-2574

49. Zhang K, Wang W, Liu Y, Guo A, Yang D: Let-7b acts as a tumor suppressor in osteosarcoma via targeting IGF1R. Oncol Lett 2019, $17: 1646-1654$

50. Wang YC, Chen YL, Yuan RH, Pan HW, Yang WC, Hsu HC, Jeng YM: Lin-28B expression promotes transformation and invasion in human hepatocellular carcinoma. Carcinogenesis 2010, 31: $1516-1522$

51. Fulton AB, Hansen RM, Moskowitz A: Development of rod function in term born and former preterm subjects. Optom Vis Sci 2009, 86: E653-E658

52. Hamilton R, Bradnam MS, Dudgeon J, Mactier H: Maturation of rod function in preterm infants with and without retinopathy of prematurity. J Pediatr 2008, 153:605-611

53. Sapieha P: Eyeing central neurons in vascular growth and reparative angiogenesis. Blood 2012, 120:2182-2194

54. Kensler TW, Wakabayashi N, Biswal S: Cell survival responses to environmental stresses via the Keap1-Nrf2-ARE pathway. Annu Rev Pharmacol Toxicol 2007, 47:89-116

55. Gambino V, De Michele G, Venezia O, Migliaccio P, Dall'Olio V, Bernard L, Minardi SP, Della Fazia MA, Bartoli D, Servillo G, Alcalay M, Luzi L, Giorgio M, Scrable H, Pelicci PG, Migliaccio E: Oxidative stress activates a specific p53 transcriptional response that regulates cellular senescence and aging. Aging Cell 2013, 12:435-445

56. El-Deiry WS: The role of p53 in chemosensitivity and radiosensitivity. Oncogene 2003, 22:7486-7495

57. Chavala SH, Kim Y, Tudisco L, Cicatiello V, Milde T, Kerur N, Claros N, Yanni S, Guaiquil VH, Hauswirth WW, Penn JS, Rafii S, De Falco S, Lee TC, Ambati J: Retinal angiogenesis suppression through small molecule activation of p53. J Clin Invest 2013, 123: $4170-4181$

58. Hussain SP, Harris CC: p53 biological network: at the crossroads of the cellular-stress response pathway and molecular carcinogenesis. J Nippon Med Sch 2006, 73:54-64

59. Qian Y, Chen X: Senescence regulation by the p53 protein family. Methods Mol Biol 2013, 965:37-61

60. Gravina S, Vijg J: Epigenetic factors in aging and longevity. Pflugers Arch 2010, 459:247-258

61. Astanehe A, Arenillas D, Wasserman WW, Leung PC, Dunn SE, Davies BR, Mills GB, Auersperg N: Mechanisms underlying p53 regulation of PIK3CA transcription in ovarian surface epithelium and in ovarian cancer. J Cell Sci 2008, 121:664-674

62. Werner H, Sarfstein R, LeRoith D, Bruchim I: Insulin-like growth factor 1 signaling axis meets p53 genome protection pathways. Front Oncol 2016, 6:159

63. Childs BG, Baker DJ, Kirkland JL, Campisi J, van Deursen JM: Senescence and apoptosis: dueling or complementary cell fates? EMBO Rep 2014, 15:1139-1153

64. Denoyelle C, Abou-Rjaily G, Bezrookove V, Verhaegen M, Johnson TM, Fullen DR, Pointer JN, Gruber SB, Su LD, Nikiforov MA, Kaufman RJ, Bastian BC, Soengas MS: Anti-oncogenic role of the endoplasmic reticulum differentially activated by mutations in the MAPK pathway. Nat Cell Biol 2006, 8:1053-1063

65. Zhang Z, Zhang L, Zhou L, Lei Y, Zhang Y, Huang C: Redox signaling and unfolded protein response coordinate cell fate decisions under ER stress. Redox Biol 2018, [Epub ahead of print] doi:10.1016/ j.redox.2018.11.005

66. Nakamura R, Sene A, Santeford A, Gdoura A, Kubota S, Zapata N, Apte RS: IL10-driven STAT3 signalling in senescent macrophages 
promotes pathological eye angiogenesis. Nat Commun 2015, 6: 7847

67. Hong HS, Kim S, Nam S, Um J, Kim YH, Son Y: Effect of substance $\mathrm{P}$ on recovery from laser-induced retinal degeneration. Wound Repair Regen 2015, 23:268-277

68. Nunes DN, Dias-Neto E, Cardo-Vila M, Edwards JK, Dobroff AS, Giordano RJ, Mandelin J, Brentani HP, Hasselgren C, Yao VJ, Marchio S, Pereira CA, Passetti F, Calin GA, Sidman RL, Arap W, Pasqualini R: Synchronous down-modulation of miR-17 family members is an early causative event in the retinal angiogenic switch. Proc Natl Acad Sci U S A 2015, 112:3770-3775

69. Liu CH, Sun Y, Li J, Gong Y, Tian KT, Evans LP, Morss PC, Fredrick TW, Saba NJ, Chen J: Endothelial microRNA-150 is an intrinsic suppressor of pathologic ocular neovascularization. Proc Natl Acad Sci U S A 2015, 112:12163-12168

70. Ertekin S, Yildirim O, Dinc E, Ayaz L, Fidanci SB, Tamer L: Evaluation of circulating miRNAs in wet age-related macular degeneration. Mol Vis 2014, 20:1057-1066

71. Kovacs B, Lumayag S, Cowan C, Xu S: MicroRNAs in early diabetic retinopathy in streptozotocin-induced diabetic rats. Invest Ophthalmol Vis Sci 2011, 52:4402-4409

72. Werner H, Karnieli E, Rauscher FJ, LeRoith D: Wild-type and mutant p53 differentially regulate transcription of the insulin-like growth factor I receptor gene. Proc Natl Acad Sci U S A 1996, 93: $8318-8323$

73. Mellios N, Woodson J, Garcia RI, Crawford B, Sharma J, Sheridan SD, Haggarty SJ, Sur M: beta2-Adrenergic receptor agonist ameliorates phenotypes and corrects microRNA-mediated IGF1 deficits in a mouse model of Rett syndrome. Proc Natl Acad Sci U S A 2014, 111:9947-9952

74. Winter J, Jung S, Keller S, Gregory RI, Diederichs S: Many roads to maturity: microRNA biogenesis pathways and their regulation. Nat Cell Biol 2009, 11:228-234

75. Jun-Hao ET, Gupta RR, Shyh-Chang N: Lin28 and let-7 in the metabolic physiology of aging. Trends Endocrinol Metab 2016, 27:132-141
76. Wai SG, Rozance PJ, Wesolowski SR, Hay WW Jr, Brown LD: Prolonged amino acid infusion into intrauterine growth restricted fetal sheep increases leucine oxidation rates. Am J Physiol Endocrinol Metab 2018, 315:E1143-E1153

77. Holzenberger M, Dupont J, Ducos B, Leneuve P, Geloen A, Even PC, Cervera P, Le Bouc Y: IGF-1 receptor regulates lifespan and resistance to oxidative stress in mice. Nature 2003, 421: 182-187

78. Kenyon C, Chang J, Gensch E, Rudner A, Tabtiang R: A C. elegans mutant that lives twice as long as wild type. Nature 1993, 366:461-464

79. Soetikno BT, Yi J, Shah R, Liu W, Purta P, Zhang HF, Fawzi AA: Inner retinal oxygen metabolism in the 50/10 oxygen-induced retinopathy model. Sci Rep 2015, 5:16752

80. Punglia RS, Lu M, Hsu J, Kuroki M, Tolentino MJ, Keough K, Levy AP, Levy NS, Goldberg MA, D’Amato RJ, Adamis AP: Regulation of vascular endothelial growth factor expression by insulin-like growth factor I. Diabetes 1997, 46:1619-1626

81. Blaauwgeers HG, Holtkamp GM, Rutten H, Witmer AN, Koolwijk P, Partanen TA, Alitalo K, Kroon ME, Kijlstra A, van Hinsbergh VW, Schlingemann RO: Polarized vascular endothelial growth factor secretion by human retinal pigment epithelium and localization of vascular endothelial growth factor receptors on the inner choriocapillaris: evidence for a trophic paracrine relation. Am J Pathol 1999, 155:421-428

82. Hellstrom A, Engstrom E, Hard AL, Albertsson-Wikland K, Carlsson B, Niklasson A, Lofqvist C, Svensson E, Holm S, Ewald U, Holmstrom G, Smith LE: Postnatal serum insulin-like growth factor I deficiency is associated with retinopathy of prematurity and other complications of premature birth. Pediatrics 2003, 112:1016-1020

83. Saint-Geniez M, Kurihara T, Sekiyama E, Maldonado AE, D'Amore PA: An essential role for RPE-derived soluble VEGF in the maintenance of the choriocapillaris. Proc Natl Acad Sci U S A 2009, 106:18751-18756

84. Hansen RM, Tavormina JL, Moskowitz A, Fulton AB: Effect of retinopathy of prematurity on scotopic spatial summation. Invest Ophthalmol Vis Sci 2014, 55:3311-3313 